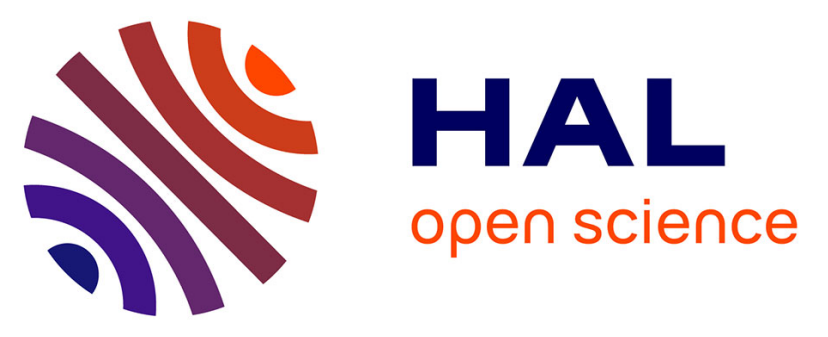

\title{
Problem of elastic anisotropy and stacking faults in stress analysis using multireflection grazing-incidence X-ray diffraction
}

Marianna Marciszko, Andrzej Baczmanski, Wrobel Miroslaw, Wilfried Seiler, Chedly Braham, Sebastian Wronski, Roman Wawszczak

\section{To cite this version:}

Marianna Marciszko, Andrzej Baczmanski, Wrobel Miroslaw, Wilfried Seiler, Chedly Braham, et al.. Problem of elastic anisotropy and stacking faults in stress analysis using multireflection grazing-incidence X-ray diffraction. Journal of Applied Crystallography, 2015, 48, pp.492-509. 10.1107/S1600576715002666 . hal-01179307

\section{HAL Id: hal-01179307 \\ https://hal.science/hal-01179307}

Submitted on 26 Aug 2015

HAL is a multi-disciplinary open access archive for the deposit and dissemination of scientific research documents, whether they are published or not. The documents may come from teaching and research institutions in France or abroad, or from public or private research centers.
L'archive ouverte pluridisciplinaire HAL, est destinée au dépôt et à la diffusion de documents scientifiques de niveau recherche, publiés ou non, émanant des établissements d'enseignement et de recherche français ou étrangers, des laboratoires publics ou privés. 


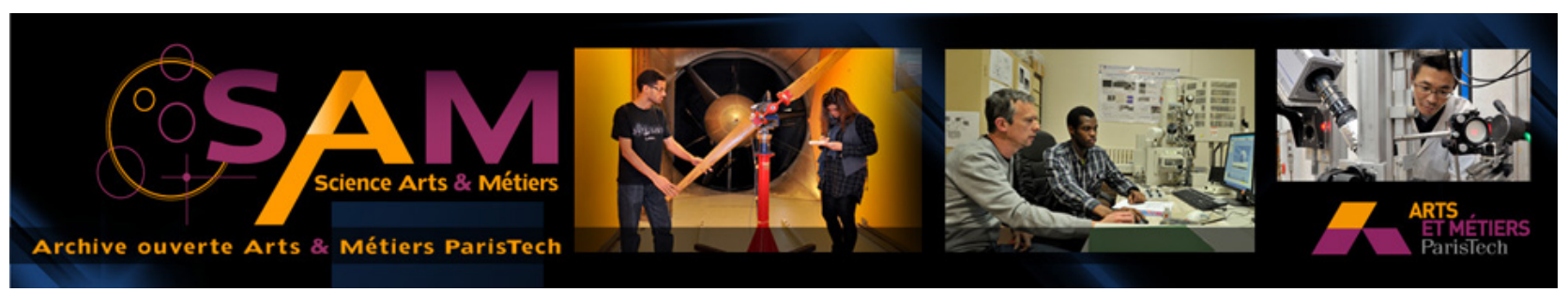

Science Arts \& Métiers (SAM)

is an open access repository that collects the work of Arts et Métiers ParisTech researchers and makes it freely available over the web where possible.

This is an author-deposited version published in: http://sam.ensam.eu Handle ID: .http://hdl.handle.net/10985/9846

\section{To cite this version :}

Marianna MARCISZKO, Andrzej BACZMANSKI, Wrobel MIROSAW, Chedly BRAHAM, Sebastian WRONSKI, Roman WAWSZCZAK - Problem of elastic anisotropy and stacking faults in stress analysis using multireflection grazing-incidence X-ray diffraction - Journal of Applied Crystallography - Vol. 48, p.492-509-2015 


\title{
Problem of elastic anisotropy and stacking faults in stress analysis using multireflection grazing- incidence $\mathrm{X}$-ray diffraction
}

\author{
Marianna Marciszko, ${ }^{\text {a }}$ Andrzej Baczmański, ${ }^{\text {a* }}$ Mirosław Wróbel, ${ }^{\mathrm{b}}$ Wilfrid Seiler, \\ Chedly Braham, ${ }^{\mathrm{c}}$ Sebastian Wroński ${ }^{\mathrm{a}}$ and Roman Wawszczak ${ }^{\mathrm{a}}$ \\ aAGH, University of Science and Technology, Faculty of Physics and Applied Computer Science, \\ Aleja Mickiewicza 30, Kraków, 30-059, Poland, 'bAGH, University of Science and Technology, \\ Faculty of Metals Engineering and Industrial Computer Science, Aleja Mickiewicza 30, Kraków, 30- \\ 059, Poland, and ${ }^{\mathbf{C}}$ Arts et Métiers-ParisTech, Laboratoire Procédés et Ingénierie en Mécanique et \\ Matériaux, CNRS UMR 8006, 151 Boulevard de I'Hôpital, Paris, 75013, France. Correspondence \\ e-mail: andrzej.baczmanski@fis.agh.edu.pl
}

\begin{abstract}
Multireflection grazing-incidence X-ray diffraction (MGIXD) was used to determine the stress- and strain-free lattice parameter in the surface layer of mechanically treated (polished and ground) tungsten and austenitic steel. It was shown that reliable diffraction stress analysis is possible only when an appropriate grain interaction model is applied to an anisotropic sample. Therefore, verification of the X-ray stress factors (XSFs) was accomplished by measuring relative lattice strains during an in situ tensile test. The results obtained using the MGIXD and standard methods ( $\chi$ and $\omega$ geometries) show that the Reuss and free-surface grain interaction models agree with the experimental data. Moreover, a new interpretation of the MGIXD results was proposed and applied for the first time to measure the probability of stacking faults as a function of penetration depth for a polished and ground austenitic sample. The XSF models verified in the tensile test were used in the analysis of residual stress components.
\end{abstract}

\section{Introduction}

Stress state is a characteristic feature of materials which, along with microstructure and texture, influences material properties. Thus, stress analysis is of great significance and has become an important part of materials science. Both the magnitude and the spatial distribution of residual stress (i.e. stress remaining in a material after different treatments or production techniques, such as casting, film deposition etc.) influence the physical properties of solids and play an important role in such processes as stress corrosion, damage (Noyan \& Cohen, 1987; Hauk, 1997; Reimers et al., 2008), plastic deformation (Dakhlaoui et al., 2006; Wroński et al., 2007), recovery and recrystallization (Humphreys et al., 2004; Wawszczak et al., 2011).

The properties of a polycrystalline material are never homogenous. Important heterogeneities of crystal structure, microstructure and residual stress are expected close to the sample surface, especially in the case of mechanically treated materials or deposited coatings (Noyan \& Cohen, 1987; Ruppersberg et al., 1989; Hauk, 1997; Reimers et al., 2008). Accordingly, methods enabling nondestructive characterization of a material as a function of depth under the surface are very important. One of these is the diffraction method, which enables direct measurements of strains in precisely defined sampling volumes in the material. Diffraction is frequently used for the measurement of lattice elastic deformation and distortion (i.e. macrostrains and microstrains) determined from the displacement and broadening of the diffraction peak (Noyan \& Cohen, 1987; Hauk, 1997; Reimers et al., 2008; Warren, 1990). In principle, the stress present in the nearsurface volume can be measured using the standard X-ray $\sin ^{2} \psi$ method. In this approach, interplanar spacings are determined using a single $h \mathrm{kl}$ reflection for different orientations of the scattering vector with respect to the sample (Noyan \& Cohen, 1987; Hauk, 1997; Reimers et al., 2008). However, this method is not recommended for the analysis of depth-dependent stress states because the penetration depth of X-ray radiation varies significantly during measurement. Therefore, a geometry based on the grazing-incidence X-ray diffraction (GIXD) method has been applied to measure residual stress (Predecki et al., 1993; Genzel et al., 1999; Skrzypek \& Baczmański, 2001; Skrzypek et al., 2001; Baczmański et al., 2004; Welzel et al., 2005; Marciszko, Baczmański, Wierzbanowski et al., 2013).

GIXD geometry is based on nonsymmetrical diffraction and performed for a small incidence angle ( $\alpha$, the angle between the incident beam and sample surface). In this case, the penetration of X-ray radiation (limited by the absorption of radiation in the studied material) depends mostly on the 
long path of the incident beam. For a constant value of the $\alpha$ angle, the penetration depth of X-ray radiation is well defined and does not change during the experiment; however, in order to perform stress measurements, the orientation of the scattering vector must be varied. Different methods have been proposed to do this (Welzel et al., 2005):

(a) a single-reflection and single-wavelength method (only one reflection and one wavelength), in which the sample must be rotated so that $\alpha$ is constant but the orientation of the scattering vector changes with respect to the sample (Genzel, 1994; van Acker et al., 1994; Genzel et al., 1999; Kumar et al., 2006; Erbacher et al., 2007);

(b) a single-reflection and multiple-wavelength method (one reflection for different wavelengths), in which appropriate wavelengths and $\alpha$ angles must be found to fulfil the condition of a constant penetration depth for different orientations of the scattering vector (Predecki et al., 1993);

(c) a multiple-reflection and single-wavelength method (MGIXD), in which many reflections for the same wavelength are measured and the $\alpha$ angle is fixed during measurements (the orientation of the scattering vector changes owing to $2 \theta$ variation; Skrzypek et al., 2001; Skrzypek \& Baczmański, 2001; Baczmański et al., 2004; Marciszko, Baczmański, Wróbel et al., 2013).

To study the stress state in a polycrystalline material, one must determine the stress from the lattice strains measured using the X-ray elastic constants (XECs) or, more generally, the X-ray stress factors (XSFs). ${ }^{\mathbf{1}}$ The simplest models, i.e. the Voigt (1928) and Reuss (1929) methods for calculation of XSFs, are based on the hypothesis of strain or stress homogeneity in the volume under consideration. These models have been applied to quasi-isotropic and textured polycrystalline materials as well (Dölle, 1979; Dölle \& Cohen, 1980; Barral et al., 1987; Brakman, 1987; Baczmański et al., 1994; Schuman et al., 1994, Welzel et al., 2005).

In more advanced models, the interaction between grains is taken into account in the calculations. For example, in the Kröner (1961) method the grain is approximated by an ellipsoidal inclusion (Eshelby, 1957) embedded in a homogenous and isotropic medium. Moreover, two approaches have been proposed to take into account the direction-dependent interaction between grains. The first was proposed by Witt \& Vook (1968) and developed by van Leeuwen et al. (1999) and Welzel et al. (2003) for columnar grains in the surface layer. It was assumed that these grains, with dimensions equal to the thickness of the film, exhibit the same in-plane strain (a Voigttype behaviour), whereby they can deform freely in a direction perpendicular to the surface (a Reuss-type behaviour). Another model, called the free-surface model, was proposed by Baczmański et al. (2008) for grains placed close to the sample surface. As in the Vook-Witt model, it was assumed that these grains can freely deform in the direction normal to the surface (Reuss-type behaviour), while in-plane interaction

\footnotetext{
${ }^{\mathbf{1}}$ Further details on XSF models are given in the supporting information, which is available from the IUCr electronic archives (Reference: NB5129). For additional literature related to this material, see Bollenrath et al. (1967), Lipinski \& Berveiller (1989) and Matthies \& Humbert (1995).
}

is approximated by a self-consistent model (Kröner-type behaviour). It should be underlined that the latter approximation describes the interaction occurring between grains in the near-surface gauge volume penetrated by X-rays, especially in cases where the MGIXD method is used. This is why the free-surface model was tested in this study.

In this paper, the MGIXD method is developed in order to gain new information concerning the variation with depth of the crystal structure and microstructure in samples exhibiting stress in their surface layers. A new methodology for simultaneous determination of stress, the strain-free lattice parameter $a_{0}$ and stacking fault probability was developed and applied to study the mechanically treated surfaces of stainless austenitic steel 316L with a low value of stacking fault energy and face-centred cubic (f.c.c.) structure. These characteristics cannot be obtained using other experimental methods applied for stress measurement (e.g. hole drilling, ultrasonic or other diffraction methods). It must be underlined that the developments of the MGIXD method presented here are possible only if the anisotropy of XSFs for different $h k l$ reflections and in different directions with respect to the sample is known. Therefore, the first part of this study concerns the experimental verification of different models for XSF calculation. The results obtained in this study enabled us to choose the best model to use for calculation of XSFs and simultaneously to identify the type of interaction occurring between grains located close to the sample surface.

\section{Principles of multireflection grazing-incidence X-ray diffraction}

In the standard $\sin ^{2} \psi$ method, the interplanar spacings $\langle d(\phi, \psi)\rangle_{\{h k l\}}$ are determined from diffraction peak positions measured for different $\psi$ angles and the constant $\phi$ angle describing the scattering vector's orientation with respect to the sample (Fig. 1). The $\langle d(\phi, \psi)\rangle_{\{h k l\}}$ spacings are measured along the direction of the scattering vector using the single reflection $h k l$ (Noyan \& Cohen, 1987; Hauk, 1997).

As has been shown (see e.g. Welzel et al., 2005), the $\psi$ angle can be changed in two different ways: by tilting the diffraction

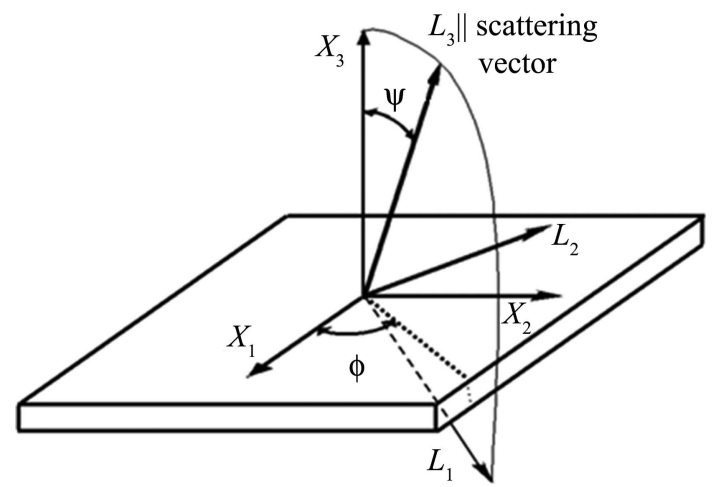

Figure 1

Orientation of the scattering vector with respect to sample frame $\mathbf{X}$. The $\psi$ and $\phi$ angles define the orientation of the $\mathbf{L}$ coordinate system (where the $L_{3}$ axis is parallel to the scattering vector and the $L_{2}$ axis lies in the plane of the sample surface). 
plane ( $\chi$ geometry) or by rotating both incident and diffracted beams in a diffraction plane perpendicular to the sample surface ( $\omega$ geometry). In both cases the diffraction peak for the same reflection $h k l$ is measured; thus the $2 \theta$ angle remains approximately constant (excluding small shifts caused by lattice strains). Measurements of $\langle d(\phi$, $\psi)\rangle_{\{h k l\}}$ versus $\sin ^{2} \psi$ functions can be repeated for different $\phi$ angles.

The standard method for stress measurement is not suitable for the analysis of depth-dependent stress states, because the penetration depth of the X-ray radiation varies significantly during measurement when the orientations of both the incident and the reflected beams are varied. The effective penetration depth (i.e. the depth at which $1-1 / e$ of the incident beam intensity is absorbed) can be calculated for the $\chi$ and $\omega$ geometries (Welzel et al., 2005):

$$
\begin{gathered}
\chi \text { geometry: } \tau=\frac{\cos \psi \sin \theta}{2 \mu}, \\
\omega \text { geometry: } \tau=\frac{\sin ^{2} \theta-\sin ^{2} \psi}{2 \mu \sin \theta \cos \psi},
\end{gathered}
$$

where $\mu$ is the linear attenuation coefficient.

Multireflection grazing-incidence X-ray diffraction (MGIXD) geometry, also called multiple $h k l$ grazing incidence, is characterized by a small and constant incidence angle $\alpha$ and different orientations of the scattering vector (variable $2 \theta_{\{h k l\}}$ angle for a constant wavelength; see Fig. 2) given by the equation (van Acker et al., 1994; Skrzypek \& Baczmański, 2001)

$$
\psi_{\{k h l\}}=\theta_{\{h k l\}}-\alpha,
$$

where $2 \theta_{\{h k l\}}$ are the diffraction angles corresponding to those reflections $h k l$ for which diffraction peaks are measured. In this geometry, the diffraction plane (defined by the incident and diffracted beams) is always perpendicular to the sample surface. Measurements of interplanar spacings are performed in the near-surface volume, which is limited by the radiation absorption and effective penetration depth of the X-ray beam in the studied material as defined by the formula (Baczmański et al., 2003)

$$
\tau=\left[\frac{\mu}{\sin \alpha}+\frac{\mu}{\sin \left(2 \theta_{\{h k l\}}-\alpha\right)}\right]^{-1} .
$$

As shown in Fig. 3, in the case of the MGIXD method, the penetration depth is almost constant for a wide range of $\sin ^{2} \psi$, and significant variation of $\tau$ versus $\sin ^{2} \psi$ occurs for both standard methods (i.e. for $\omega$ and $\chi$ geometries). When the $\alpha$ angle in the MGIXD method is small, the path of the incident beam is long $[a(z)>>b(z)$, where $z$ is the depth under the surface defined in Fig. 2] and equation (4) can be simplified:

Figure 2

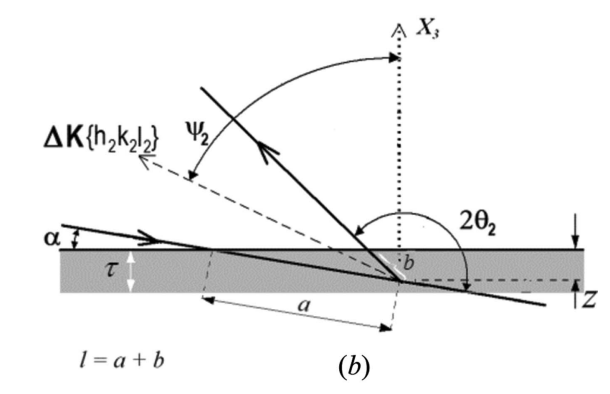

(a)

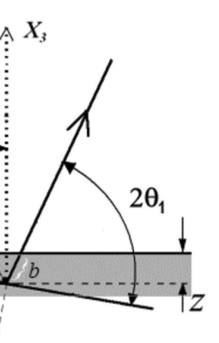
orientation of the scattering vector $\Delta \mathbf{K}_{\{h k l\}}$ is characterized by the angle $\psi_{\{h k l\}}$. The depth under the surface and penetration depth are denoted by $z$ and $\tau$, respectively.

$$
\tau=(\sin \alpha) / \mu
$$

Here $\tau$ does not depend on the $\theta_{\{h k l\}}$ angle and is constant when interplanar spacings are measured for different orientations of the scattering vector described by the $\psi_{\{h k l\}}$ angle. It can be concluded that stress measurement can be performed for a well defined penetration depth $\tau$ by using the MGIXD method. Moreover, it is possible to perform measurements at different depths below the sample surface by choosing appropriate values of the $\alpha$ angle [see equation (5)].

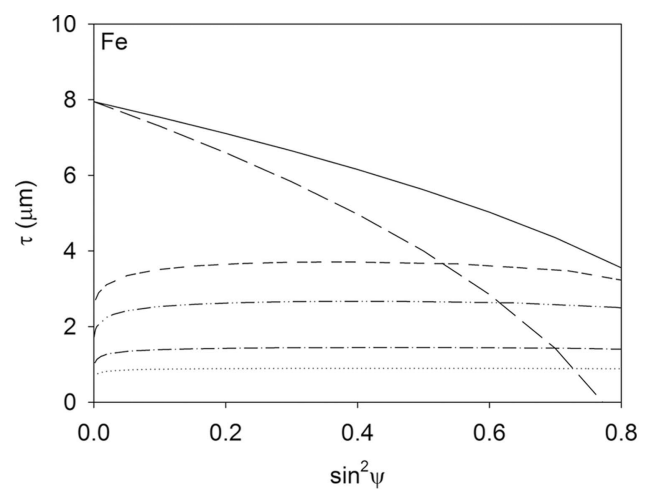

(a)

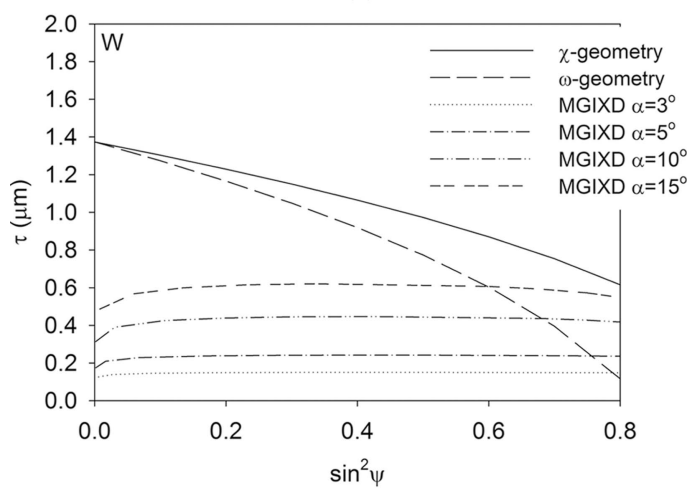

(b)

Figure 3

Penetration depth as a function of $\sin ^{2} \psi$ calculated from equations (1), (2) and (4) for (a) austenitic steel $\left(\mu=554 \mathrm{~cm}^{-1}\right.$, Fe $K \alpha_{1}$ radiation) and (b) polished tungsten $\left(\mu=3311 \mathrm{~cm}^{-1}, \mathrm{Cu} K \alpha_{1}\right.$ radiation). In the standard geometry, the $311\left(2 \theta=123.5^{\circ}\right)$ and $321\left(2 \theta=131.3^{\circ}\right)$ reflections were used for austenitic steel and tungsten, respectively. 
Analogously to the standard method (Noyan \& Cohen, 1987; Hauk, 1997; Welzel et al., 2005), stress can be determined from interplanar spacings measured for different $\psi_{\{h k l\}}$ (i.e. a constant $\alpha$ angle and various $\theta_{\{h k l\}}$ angles). In the multireflection method, the so-called equivalent lattice parameters $\langle a(\phi, \psi, \tau)\rangle_{\{h k l\}}^{\mathrm{cal}}$ are calculated and expressed using the macrostress $\sigma_{i j}(\tau)$ and the strain-free $a_{0}$ lattice constant (Dölle \& Hauk, 1978; Dölle, 1979; Baczmański et al., 2003; Skrzypek et al., 2001; Ortner, 2005, 2006):

$$
\langle a(\phi, \psi, \tau)\rangle_{\{h k l\}}^{\mathrm{cal}}=\left[F_{i j}(h k l, \phi, \psi) \sigma_{i j}(\tau)\right] a_{0}+a_{0},
$$

where $F_{i j}(h k l, \phi, \psi)$ are the XSFs and $\phi$ describes the sample rotation around the surface normal (Fig. 1), while $\psi_{\{h k l\}}$ depends on the diffraction angle for a given $h k l$ reflection [cf. Fig. 2 and equation (3)]. In the equations above and below, the Einstein summation convention is applied for the repeated indices. For cubic crystal structure, experimental values of equivalent lattice parameters are calculated from the measured interplanar spacings $\langle d(\phi, \psi, \tau)\rangle_{\{h k l\}}$ :

$$
\langle a(\phi, \psi, \tau)\rangle_{\{h k l\}}^{\exp _{0}}=\langle d(\phi, \psi, \tau)\rangle_{\{h k l\}}\left(h^{2}+k^{2}+l^{2}\right)^{1 / 2} .
$$

In the case of the general stress state, and assuming ${ }^{2}$ that $\sigma_{33}(\tau)=0$, the other components of the stress tensor and $a_{0}$ parameter can be determined by fitting $\langle a(\phi, \psi, \tau)\rangle_{\{h k l\}}^{\text {cal }}$ [expressed by equation (6)] to the experimental values yielded by equation (7). The components of the stress tensor and $a_{0}$ parameter are independent values varied in the least-squares fitting procedure, while the values of $F_{i j}(h k l, \phi, \psi)$ must be known from the model or a previous measurement. The calculations performed in this study were based on minimizing the merit function called $\chi^{2}$, defined as (Press et al., 1989)

$$
\chi^{2}=\frac{1}{N-M} \sum_{n=1}^{N}\left[\frac{\left\langle a\left(\phi_{n}, \psi_{n}\right)\right\rangle_{\{h k l\}}^{\exp }-\left\langle a\left(\phi_{n}, \psi_{n}\right)\right\rangle_{\{h k l\}}^{\mathrm{cal}}}{\delta_{n}}\right]^{2},
$$

where $\left\langle a\left(\phi_{n}, \psi_{n}\right)\right\rangle_{\{h k l\}}^{\exp }$ and $\left\langle a\left(\phi_{n}, \psi_{n}\right)\right\}_{\{h k l\}}^{\mathrm{cal}}$ are the experimental and calculated lattice parameters, $\delta_{n}=\delta_{n}\left[\langle a(\phi, \psi)\rangle_{\{h k l\}}\right]$ is the measurement uncertainty (standard deviation) of $\left\langle a\left(\phi_{n}, \psi_{n}\right)\right\rangle_{\{211\}}^{\exp }$ for the $n$th measurement, and $N$ and $M$ are the number of measured points and fitting parameters, respectively.

Applying the method described above to the sets of interplanar spacings measured for different incident $\alpha$ angles, the dependence of stress and the $a_{0}$ lattice parameter on penetration depth $\tau$ can be determined. With additional assumptions or approximations, the variations of these quantities versus real depth under the sample surface can also be calculated [for example, the inverse Laplace transform was used by Genzel et al. (1999) and Klaus \& Genzel (2013)]; however, this data treatment is beyond the scope of the present work and only variation versus penetration depth is presented in this paper.

\footnotetext{
${ }^{2}$ This assumption results from the relaxation of surface normal stress in the shallow volume of the material penetrated by X-rays and has been commonly used in other methods of stress determination using X-ray diffraction (Hauk, 1997).
}

\section{The influence of elastic anisotropy and stacking faults on the interpretation of MGIXD results}

It should be underlined that, in the case of anisotropic singlecrystal elastic constants, reliable stress analysis is possible only when an appropriate grain interaction model is applied to the calculation of the XECs or XSFs (four different models are briefly recalled in the supporting information). In the present

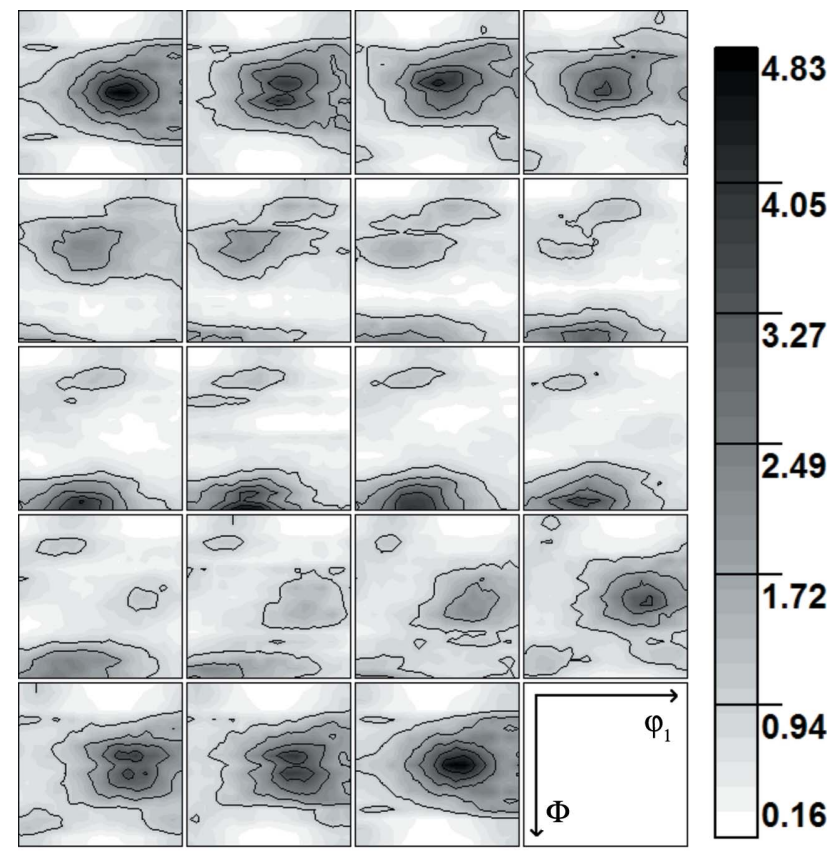

(a)
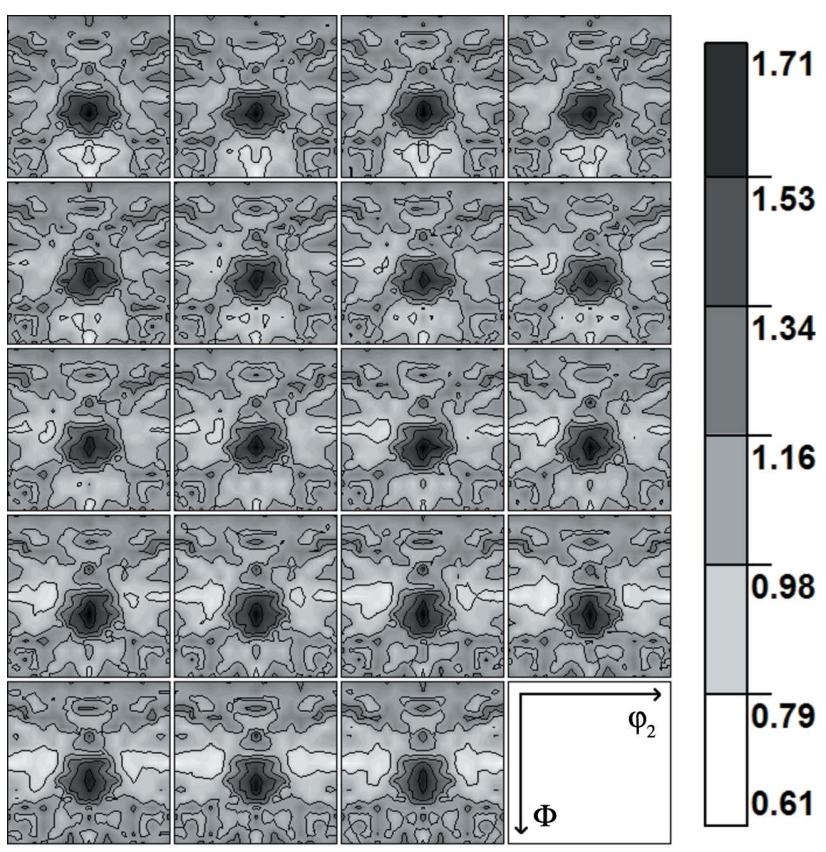

(b)

Figure 4

ODFs determined for $(a)$ austenitic stainless steel and $(b)$ polished tungsten. Sections through the basic range $0 \leq \varphi_{1}, \Phi, \varphi_{2} \leq 90^{\circ}$ of Euler space (Bunge, 1982) with a step of $5^{\circ}$ along the $\varphi_{1}$ axis for austenitic steel (a) and along the $\varphi_{2}$ axis for tungsten $(b)$ are presented. 
study, the best-fitting models to calculate the XSFs for polycrystalline materials characterized by low (tungsten) and high (austenitic stainless steel) elastic anisotropy of crystallites were identified and then applied to determine stress using the MGIXD method. For austenitic stainless steel, the XSF models were investigated during a tensile test (see $\S 4$ ); for the other samples (polished tungsten, polished and ground austenite stainless steel) the residual stress components were analysed following surface treatment (the results are shown in $\S 5)$. Moreover, in the case of mechanically treated austenitic samples, the influence of stacking faults on MGIXD measurements was taken into account for the first time. As a result, the probability of a stacking fault for $\{111\}$ planes was determined. Below is a description of the effects of elastic anisotropy and the presence of stacking faults on $\langle a(\phi, \psi)\rangle_{\{h k l\}}$ versus $\sin ^{2} \psi$ plots measured using the MGIXD method.

\subsection{Sample preparation and texture measurements}

The studied austenitic sample (AISI316L) was machined from a commercial hot-rolled and solution-annealed (at $1323 \mathrm{~K}$ ) sheet; the cylindrical tungsten sample (diameter $16 \mathrm{~mm}$, height $10 \mathrm{~mm}$ ) was produced by metallurgical powder technology followed by forging. At first, the influence of the texture function on simulated lattice strains corresponding to given macroscopic stress was studied. The $\{111\},\{200\}$ and $\{220\}$ pole figures were measured using $\mathrm{Cu} K \alpha$ radiation for hot-rolled austenitic steel (AISI316L); the $\{110\},\{200\}$ and $\{211\}$ pole figures for the polished tungsten sample were determined by $\mathrm{Mn} K \alpha$ radiation. The pole figures were measured on a standard texture goniometer, using Bragg-Brentano geometry with a point focus (a collimator with a diameter of $1.5 \mathrm{~mm}$ ). These measurements do not take depthdependent gradients of the crystallographic texture into account.

The orientation distribution functions (ODFs; see Bunge, 1982) in Fig. 4 were calculated from the experimental pole figures using the WIMV algorithm (Kallend et al., 1990). In the case of the austenitic sample, the Euler angles were defined with respect to the sample frame determined by the directions characteristic for rolling, i.e. $x_{1} \| \mathrm{TD}$ (transverse direction), $x_{2} \| \mathrm{RD}$ (rolling direction) and $x_{3} \| \mathrm{ND}$ (normal direction) (Fig. 4a). A fibre-type texture was determined in the tungsten sample; consequently the $x_{1}$ and $x_{2}$ axes were defined arbitrarily in the surface plane, while $x_{3}$ was surface

Figure 5 TD). normal (the same sample frame was later used to define the orientation of the measured stress tensor).

\subsection{Influence of elastic anisotropy on lattice strains}

This section is aimed at showing the effect of elastic anisotropy on stress analysis performed using the MGIXD method for textured materials. To demonstrate the influence of elastic anisotropy on the lattice strains, the predicted $\langle\varepsilon(\phi$, $\psi)\rangle_{\{h k l\}}$ versus $\sin ^{2} \psi$ plots corresponding to uniaxial stress $\sigma_{11}=500 \mathrm{MPa}$ are shown in Fig. 5. The lattice strains $\langle\varepsilon(\phi$, $\psi)\rangle_{\{h k l\}}$ were calculated from the equation

$$
\langle\varepsilon(\phi, \psi)\rangle_{\{h k l\}}=\frac{\langle d(\phi, \psi)\rangle_{\{h k l\}}-d_{0\{h k l\}}}{d_{0\{h k l\}}}=F_{11}(h k l, \phi, \psi) \sigma_{11},
$$

where $d_{0\{h k l\}}$ is the strain-free interplanar spacing; the XSFs were determined using four different grain interaction models (see supporting information) for the single-crystal elastic constants given in Table 1, assuming the random distribution of orientations or texture functions presented in Fig. 4.

In analysing Figs. 5(a) and 5(b), it can be concluded that in the case of elastically isotropic tungsten the same results were obtained for different models with random grain orientations as well as textures. As expected, the $\langle\varepsilon(\phi, \psi)\rangle_{\{h k l\}}$ versus $\sin ^{2} \psi$ plots are linear because the XSFs do not depend on the $h k l$ reflection ( $c f$. supporting information). Similar linear curves were obtained using the standard method when a single $h k l$

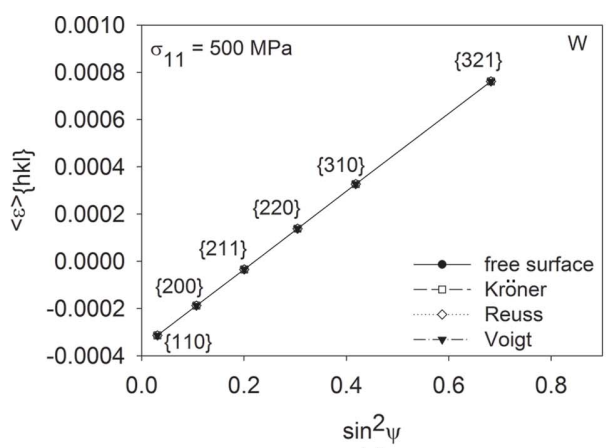

(a)

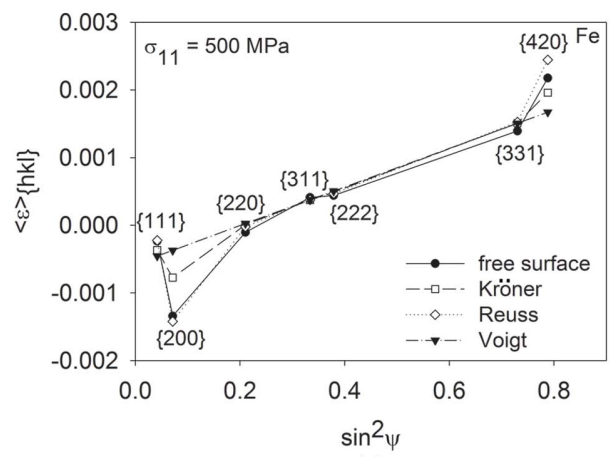

(c)

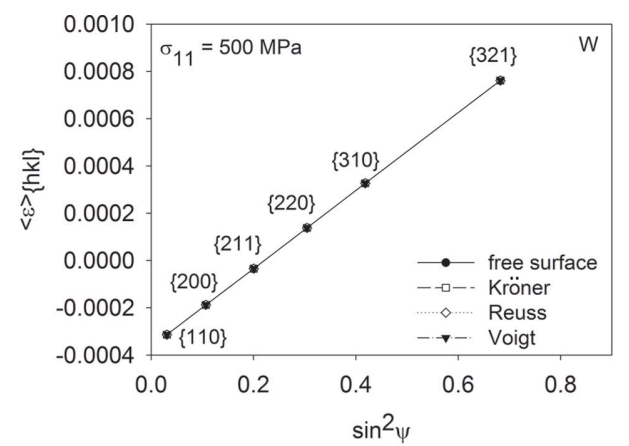

(b)

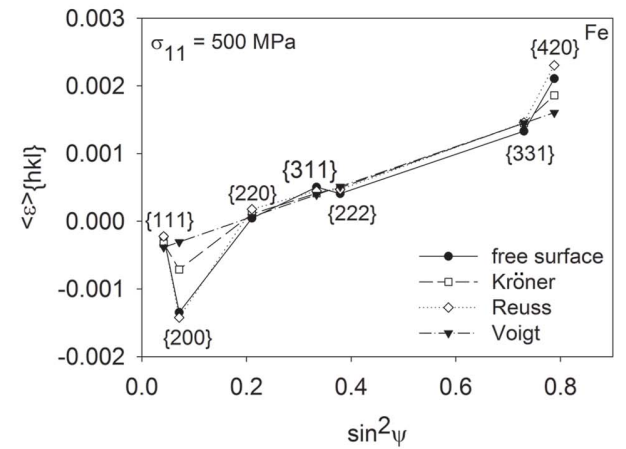

$(d)$

Lattice strains calculated for different $h k l$ reflections used in MGIXD, predicted for uniaxial tensile stress $\Sigma_{11}=500 \mathrm{MPa}$ applied to $(a),(b)$ tungsten and $(c),(d)$ austenitic steel. The XSFs were calculated using the single-crystal elastic constants given in Table 1 and assuming $(a),(c)$ random texture or $(b),(d)$ the experimental texture functions presented in Fig. 4 (in the case of cold-rolled austenite, $\sigma_{11}$ is parallel to 
Table 1

Single-crystal elastic constants $\left(c_{i j}, \mathrm{GPa}\right)$ and Zener's anisotropy factor $(A)$ for the studied materials (Simmons \& Wang, 1971).

\begin{tabular}{lccccccc}
\hline Material & $c_{11}$ & $c_{12}$ & $c_{13}$ & $c_{33}$ & $c_{44}$ & $c_{66}$ & $A$ \\
\hline Tungsten & 501 & 198 & 198 & 501 & 151 & 151 & 1 \\
Austenite (AISI316L) & 197 & 122 & 122 & 197 & 124 & 124 & 3.3 \\
\hline
\end{tabular}

reflection was used ( $c f$. Noyan \& Cohen, 1987; Hauk, 1997). The nonlinearity of the plots of $\langle\varepsilon(\phi, \psi)\rangle_{\{h k l\}}$ versus $\sin ^{2} \psi$ (Figs. $5 c$ and $5 d$ ) is caused by the significant crystal anisotropy of austenite, which leads to a strong dependence of the XSFs on the $h k l$ reflection. In this case, different models give significantly different results, i.e. the strongest nonlinearities are predicted by the Reuss model, while a linear plot is given by the Voigt model. The crystallographic texture does not change the values of the lattice strains for the studied austenitic sample significantly, as can be seen by comparing Figs. 5(c) and 5(d). It can be concluded that for both studied samples the influence of texture on the experimental results is not significant; in fact, only crystal anisotropy plays an important role in the interpretation of the stress measurements performed using the MGIXD method. Despite the minor influence of texture on the results, the ODFs were taken into account for all XSFs calculated in this work (thus the characterizations of the obtained samples are closer to the real state of the material). It can be also concluded that texture gradients which may be present in the studied materials do not significantly influence the interpretation of the measurements performed in this study.

The simulations presented in this section prove that the problem of crystal anisotropy is very important for correct interpretation of the experimental data obtained using the MGIXD method. Therefore, different models of XSF calculations must be verified by comparing them with the experimental data obtained during the in situ tensile test for the anisotropic austenitic sample.

\subsection{Influence of stacking faults on measured lattice para- meters}

Residual stress is not the only reason for the diffraction peak shift with respect to the position corresponding to the perfect lattice. Stacking faults may also be the cause (Hauk, 1997). The first studies concerning this effect were done by Paterson (1952), Warren \& Warekois (1955) and Wagner $(1957,1966)$, and the next by Velterop et al. (2000) and Jegou et al. (2013), in which it was shown that stacking faults can significantly change the position of diffraction lines. This is especially important for f.c.c. crystals with low stacking fault energy (e.g. austenitic steels). In this case, the magnitude of the peak shift is proportional to the probability of finding the stacking fault for two neighbouring $\{111\}$ planes. In the absence of second-order plastic incompatibility stress (Baczmański et al., 2003), the following can be used (following Warren \& Warekois, 1955; Wagner, 1966; Baczmański, 2005):

$$
\langle a(\phi, \psi)\rangle_{\{h k l\}}=\left[F_{i j}(h k l, \phi, \psi) \sigma_{i j}+\rho G(h k l)\right] a_{0}+a_{0},
$$

with

$$
\begin{array}{r}
G(h k l) \cong \frac{3^{1 / 2}}{4 \pi m} \sum_{m} \frac{s L_{0}}{h^{2}+k^{2}+l^{2}}, \quad L_{0}=h+k+l, \\
s=\left\{\begin{array}{ll}
+1 & \text { for } L_{0}=3 M-1, \\
0 & \text { for } L_{0}=3 M, \\
-1 & \text { for } L_{0}=3 M+1,
\end{array} \text { and } M \in \mathbb{Z} .\right.
\end{array}
$$

Here $G(h k l)$ is the coefficient reflecting relative changes in the determined interplanar spacings caused by stacking faults; the sum is calculated over all symmetrically equivalent $\{h k l\}$ planes; $m$ is the plane multiplicity; and $\rho$ is the probability of finding the deformation stacking fault for two neighbouring planes $\{111\}$.

Strictly speaking, the $\rho$ value refers to the stacking faults produced during plastic deformation (so-called deformation stacking faults). A transmission electron microscopy study confirmed that stacking faults were present in the plastically deformed stainless steel used in this study (Fig. 6), and their influence on the measured lattice parameter must be taken into account in the stress analysis. Both macrostress and stacking faults cause nonlinearities in the $\langle a(\phi, \psi)\rangle_{\{h k l\}}$ versus $\sin ^{2} \psi$ plots, i.e. a deviation of the determined $\langle a(\phi, \psi)\rangle_{\{h k l\}}$ lattice parameter from a linear dependence on $\sin ^{2} \psi$. These effects are demonstrated in Fig. 7, where the $\langle a(\phi, \psi)\rangle_{\{k k l\}}$ versus $\sin ^{2} \psi$ plots were simulated using equation (10) for applied tensile or compressive stress (see Figs. $7 a$, and $7 b$ ) or in the presence of the stacking faults (Fig. 7c). Calculations were done for experimental conditions corresponding to the austenitic sample measured using the MGIXD method with $\mathrm{Cu} K \alpha$ radiation (assumed strain-free lattice parameter $a_{0}=$ $3.595 \AA$ A incident angle $\alpha=10^{\circ}$ ). As shown in Figs. 7( $a$ ) and $7(b)$, macrostress changes the slope (illustrated by the linear regression line) as well as causing the nonlinearity of the $\langle a(\phi, \psi)\rangle_{\{h k l\}}$ versus $\sin ^{2} \psi$ curve (departure of the values from

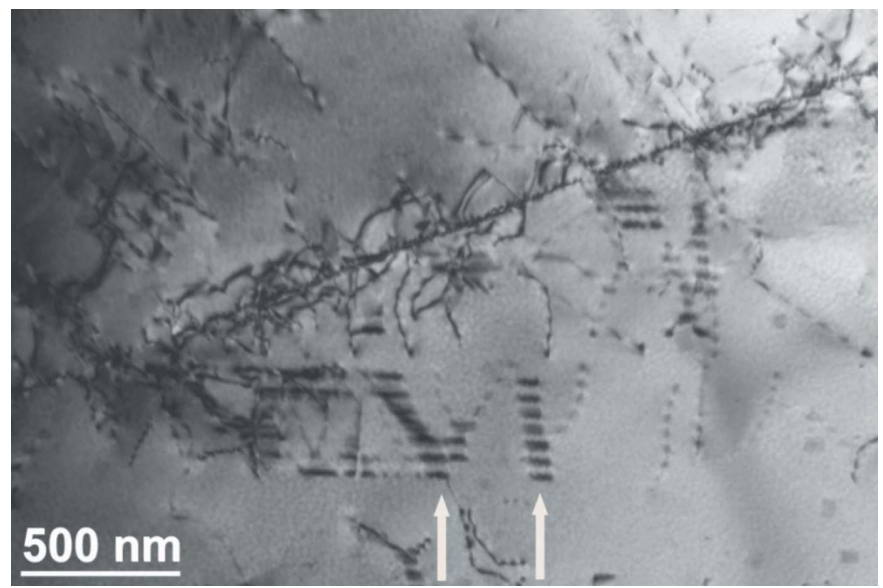

Figure 6

Stacking faults (marked by arrows) and dislocations in plastically deformed austenitic stainless steel AISI316L (transmission electron microscopy, bright field image). 
the regression line). Also, the trend of nonlinearities changes for tensile and compressive stress ( $c f$. the 200 and 111 reflections). In contrast, the stacking faults mostly increase the nonlinearity of these plots; nevertheless, the slope remains approximately constant if uniform and isotropic fault probability is assumed and all of the reflections shown in Fig. 7(c) are used in the analysis [see the linear regression line in Fig. 7(c)]. It was confirmed that the change in slope becomes more significant if one of the 200 and 111 reflections is omitted. It should be underlined that a nonzero slope caused by the presence of stacking faults can lead to an erroneous value for determined stress if the influence of the faults is not taken into account in the stress analysis or if an incorrect

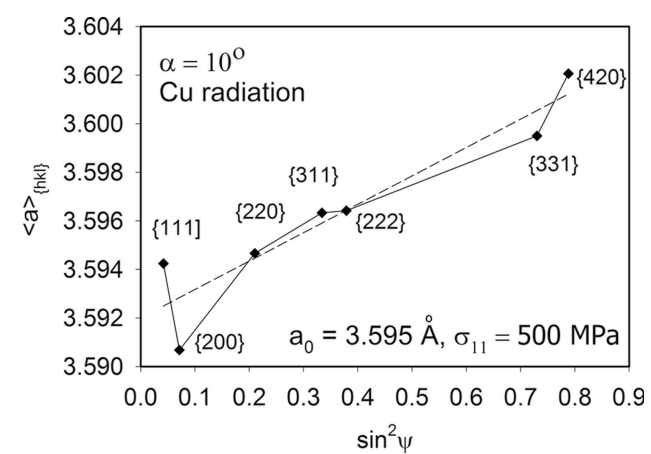

(a)

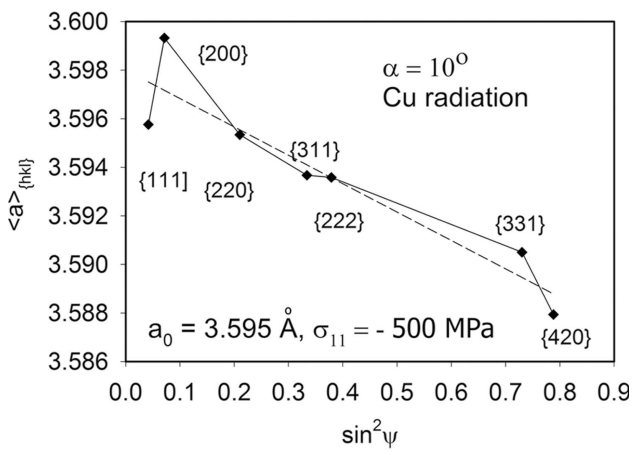

(b)

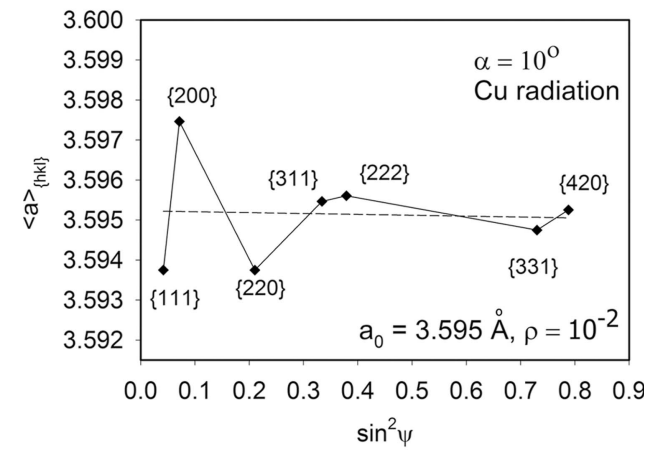

(c)

Figure 7

Simulated function $\langle a(\phi, \psi)\rangle_{\{h k l\}}$ versus $\sin ^{2} \psi$ for an austenitic sample (assuming $a_{0}=3.595 \AA, \alpha=10^{\circ}$ and $\mathrm{Cu} K \alpha$ radiation) for (a) uniaxial tensile stress $\sigma_{11}=500 \mathrm{MPa},(b)$ uniaxial compressive stress $\sigma_{11}=-500 \mathrm{MPa}$ and $(c)$ stacking fault probability $\rho=10^{-2}$. XSFs were calculated using the free-surface model with the elastic constants given in Table 1 and assuming a random texture. A dashed line is fitted to the simulated values using linear regression. model is used for the calculation of XSFs [especially in the case of the Voigt model predicting a linear $\langle a(\phi, \psi)\rangle_{\{h k l\}}$ versus $\sin ^{2} \psi$ curve]. The erroneous stress value depends strongly on the number and type of $h k l$ reflections used in the stress analysis. The aim of this study is not only to determine the stacking fault probability in a sample exhibiting residual stress, but also to take into account the effect of stacking faults on the experimental data in order to calculate the correct values of stress.

The different influences of stress and stacking faults on the $\langle a(\phi, \psi)\rangle_{\{h k l\}}$ versus $\sin ^{2} \psi$ curve enable the separation of two effects with different origins. To take both effects into account in the analysis, the $\langle a(\phi, \psi)\rangle_{\{h k l\}}$ lattice parameters expressed by equation (10) should be fitted to the experimental data using the least-squares procedure (Press et al., 1989). In the calculations, the values of stress $\sigma_{i j}$ as well as the $\rho$ parameter are varied in order to obtain the best agreement between the theoretical and the experimental nonlinear $\langle a(\phi, \psi)\rangle_{\{k k l\}}$ versus $\sin ^{2} \psi$ curves. The adjusted $\rho$ parameter is the probability of finding the stacking fault for the neighbouring planes $\{111\}$. As a result of the presented method, the stress and the probability of stacking faults can be determined simultaneously.

It should be mentioned that the idea of fitting used in this work is similar to that applied to the determination of secondorder plastic incompatibility stress, where an additional adjusting parameter was also used by Baczmański et al. (1994, 2003, 2008, 2009) and Wroński et al. (2007). In the general case of a severely deformed sample containing not only stacking faults but also significant levels of anisotropic second-order plastic incompatibility stress, an additional term should be added to equation (10) (consequently, an additional parameter responsible for this stress must be adjusted in the fitting procedure). To avoid the problem of ill-conditioned fitting, only samples exhibiting a negligible shift in the diffraction peak caused by plastic incompatibility stress were used in this study. For these samples, the nonlinearities of $\langle a(\phi, \psi)\rangle_{\{h k l\}}$ versus $\sin ^{2} \psi$ can be correctly fitted using equation (10), where elastic anisotropy (i.e. second-order stress of elastic origin) and the presence of stacking faults are taken into account. In previous studies, it was shown that monotonic deformation of textured material, e.g. uniaxial tension or cold rolling, is necessary to generate significant values of anisotropic plastic incompatibility stress causing nonlinearities of the $\langle d(\phi, \psi)\rangle_{\{h k l\}}$ versus $\sin ^{2} \psi$ plots determined using a single $h k l$ reflection (e.g. Baczmański et al., 2008, 2009; Wroński et al., 2007; Wawszczak et al., 2011). However, this does not apply to the mechanical polishing or grinding treatments performed in this study, where the deformation is not monotonic and nonlinearities of the $\langle d(\phi, \psi)\rangle_{\{h k l\}}$ versus $\sin ^{2} \psi$ plots, measured for one $h \mathrm{kl}$ reflection, are not usually generated. Moreover, tensile macrostress created during grinding has a thermal origin (as for the samples used in this study) (Malkin \& Guo, 2007), and the thermal process does not introduce secondorder incompatibility stress in one-phase cubic material (thermal expansion coefficients are isotropic for cubic crystallites). 


\section{Experimental verification of XSFs for the austenitic sample}

The correct choice of model for the calculation of XSFs is significant for materials exhibiting high elastic anisotropy. In order to select the appropriate model, either the theoretical $\langle a(\phi, \psi)\rangle_{\{h k l\}}$ versus $\sin ^{2} \psi$ curve was compared with the experimental results (Baczmański et al., 2008) or the XSFs were directly measured in situ for the sample subjected to external load (Baczmański et al., 1994). In this study, another method was used to verify model XSFs: the lattice strains were measured in situ during the tensile test in the elastic range of the deformation and the model XSFs were used to determine the stress applied to the sample. It was confirmed that the given model enables the value of the applied stress to be recalculated from the diffraction data.

The MGIXD method and the standard method ( $\chi$ mode 311 reflection) were used to measure stress in the austenitic stainless steel (AISI316L) sample under different known values of tensile uniaxial stress $\Sigma_{11}$ applied along TD. In this case, the sample frame (Fig. 1) was defined by the axes $x_{1}\left\|\mathrm{TD}, x_{2}\right\| \mathrm{RD}$ and $x_{3} \| \mathrm{ND}$. Measurements were performed on a Seifert PTS MZ VI diffractometer with Fe $K \alpha$ radiation and a parallel plate collimator (Söller collimator) in reflected beam optics (parallel beam configuration). Prior to measurement, a sample surface layer of $200 \mu \mathrm{m}$ was removed by electropolishing.

First, the values of the residual stress $\sigma_{11}^{\text {init }}$ and $\sigma_{22}^{\text {init }}$ in the TD and $\mathrm{RD}$ of the initial (nonloaded) sample were determined using both the standard ( $\chi$ mode) and MGIXD methods. With the application of analysis based on equation (6), small levels of compressive $\left[\sigma_{11}^{\text {init }} \simeq-27(24) \mathrm{MPa}\right]$ and tensile $\left[\sigma_{22}^{\text {init }} \simeq\right.$ 25 (23) $\mathrm{MPa}$ ] stress with $\alpha=20^{\circ}$ were measured [MGIXD method with $\alpha=20^{\circ}$ and Kröner-type XSFs calculated with the texture shown in Fig. 4(a)]. The differences between the results of the MGIXD and standard methods as well as between the values of stress obtained using the different models of XSFs were smaller than $10 \mathrm{MPa}$, i.e. within the uncertainty range.

In order to avoid the influence of residual stress and/or systematic errors in the determined peak positions during verification of the XSFs, measurements were performed for a nonloaded sample and a sample under applied uniaxial stress. To do this, a bone-shaped sample was machined from hotrolled austenitic steel (AISI316L) and the load was applied along TD. The relative differences between the lattice parameters for the loaded [i.e. $\left.\langle a(\phi, \psi)\rangle_{\{h k l\}}^{\text {load }}\right]$ and nonloaded samples [i.e. initial: $\left.\langle a(\phi, \psi)\rangle_{\{h k l\}}^{\text {init }}\right]$ were determined:

$$
\langle\varepsilon(\phi, \psi)\rangle_{\{h k l\}}^{\mathrm{rel}}=\frac{\langle a(\phi, \psi)\rangle_{\{h k l\}}^{\mathrm{load}}-\langle a(\phi, \psi)\rangle_{\{h k l\}}^{\mathrm{init}}}{\langle a(\phi, \psi)\rangle_{\{h k l\}}^{\mathrm{init}}} .
$$

To calculate the strain $\varepsilon$ from equation (11), the exact value of the strain-free lattice parameter is not necessary. Moreover, the effect of residual stress and/or systematic errors in the determined peak positions is avoided when the relative strain is determined (Brakman, 1988; Baczmański et al., 1994). Taking into account the reasonable assumption that the structure and number of stacking faults do not change during elastic deformation, we can also state that shifts in the $\langle a(\phi, \psi)\rangle_{\{h k l\}}^{\text {init }}$ and $\langle a(\phi, \psi)\rangle_{\{h k l\}}^{\text {load }}$ values caused by the faults are the same in both the initial and loaded samples. Therefore, the presence of stacking faults influences neither the relative strains calculated according to equation (11) nor, consequently, the results of our verification based on the relative values of the strains. It can be concluded that in the elastic (linear) deformation range the values of the relative strains determined in our experiment correspond directly to the applied stress $\Sigma_{11}$ and are not influenced either by residual macrostress and second-order stress or by stacking faults present in the initial sample.

In order to determine the stress state in the sample (corresponding directly to the applied stress $\Sigma_{11}$ ) the principal components of biaxial stress were determined using the leastsquares fitting based directly on the relationship (Baczmański et al., 1994)

$$
\langle\varepsilon(\phi, \psi)\rangle_{\{h k l\}}^{\mathrm{rel}}=F_{11}(h k l, \phi, \psi) \sigma_{11}^{\mathrm{rec}}+F_{22}(h k l, \phi, \psi) \sigma_{22}^{\mathrm{rec}},
$$

where $F_{11}(h k l, \phi, \psi)$ and $F_{22}(h k l, \phi, \psi)$ are the XSFs predicted by the given model of grain interaction and the values of $\sigma_{11}^{\text {rec }}$ and $\sigma_{22}^{\text {rec }}$ are adjusted in the fitting procedure ('rec' means that the known values of stress applied to the sample were recalculated from the diffraction data).

The main challenge of this part of the study was to verify which type of XSF enables recalculation of the value of the applied stress $\Sigma_{11}$ from the measured relative lattice strains [equation (11)] and to verify the agreement of the model results with the experimental data. The best models were selected according to two criteria:

(a) the minimum value of the goodness parameter $\chi^{2}$ :

$$
\chi^{2}=\frac{1}{N-M} \sum_{n=1}^{N}\left[\frac{\left\langle\varepsilon\left(\phi_{n}, \psi_{n}\right)\right\rangle_{\{h k l\}}^{\exp }-\varepsilon\left\langle\left(\phi_{n}, \psi_{n}\right)\right\rangle_{\{h k l\}}^{\mathrm{cal}}}{\delta_{n}}\right]^{2},
$$

where 'exp' and 'cal' denote experimental relative strain and that calculated by the fitting procedure, respectively [the other quantities are defined as in equation (8)];

(b) the minimum difference between the values of stress applied to the sample $\left(\Sigma_{11} \neq 0\right.$ and $\left.\Sigma_{22}=0\right)$ and those recalculated from the measured relative lattice strains $\left(\sigma_{11}^{\text {rec }}\right.$ and $\sigma_{22}^{\text {rec }}$ ).

Although the above criteria concern the same verification test for XSFs, an important difference between them should be emphasized. The first is more sensitive to elastic anisotropy, i.e. if the anisotropy of the XSFs is not correctly predicted by the tested model, the theoretical nonlinearities of the $\langle a(\phi, \psi)\rangle_{\{h k l\}}$ versus $\sin ^{2} \psi$ plot will not agree with the experimental ones and consequently the value of the $\chi^{2}$ parameter will increase. The second criterion verifies whether the model mean/overall elastic properties (average over many grains contributing to different $h \mathrm{kl}$ reflections) agree with the real ones. If this condition is fulfilled, the applied values of stress are correctly recalculated from the X-ray data using the model XSFs. 

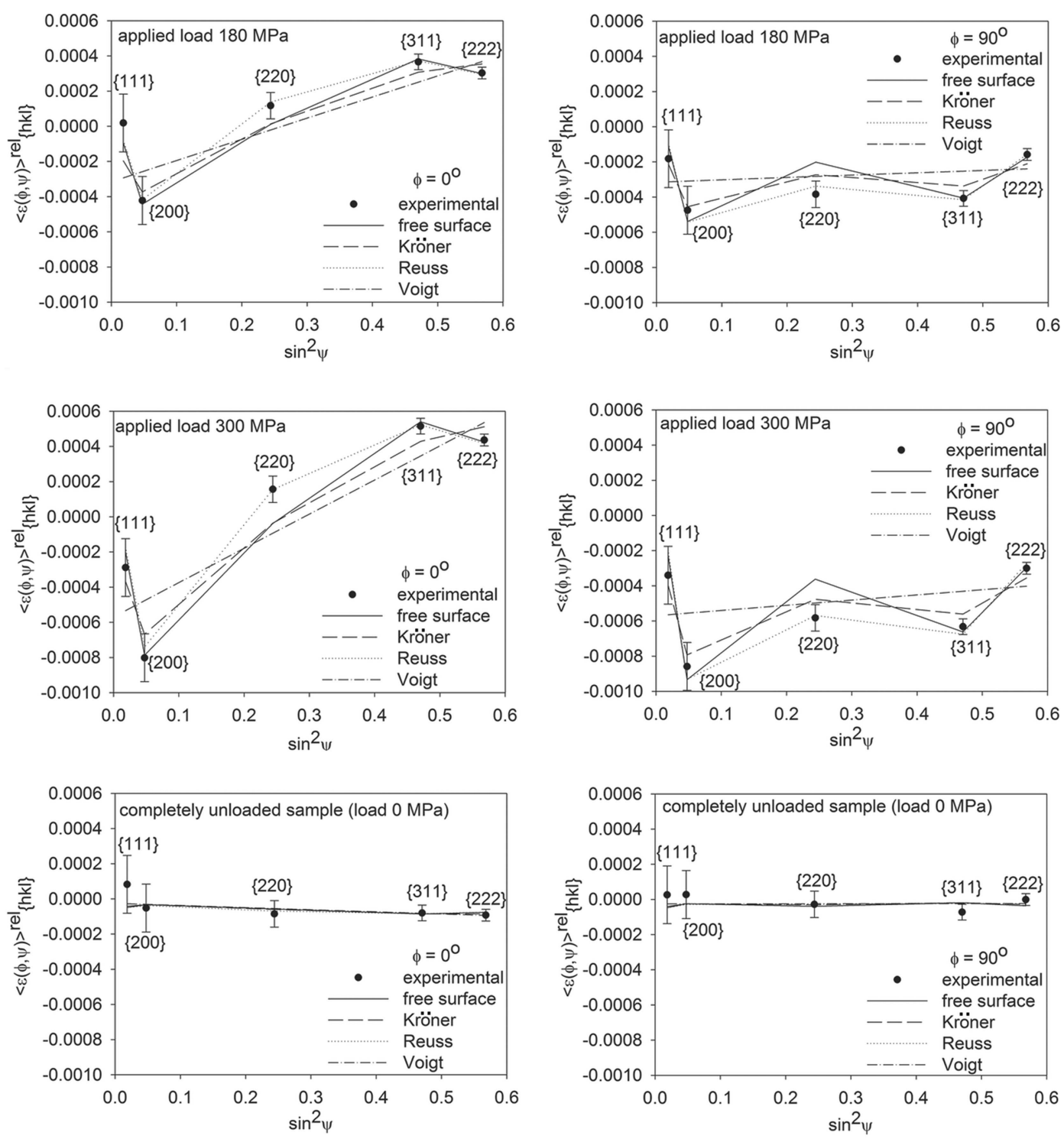

Figure 8

Relative lattice strains $\langle\varepsilon(\phi, \psi)\rangle_{\{h k l\}}^{\text {rel }}$ versus $\sin ^{2} \psi$ (incident angle $\alpha=20^{\circ}$ ) for two stages of loading and for a completely unloaded austenitic sample. Experimental results are fitted using equation (12) with XSFs calculated using the four tested models.

The models of XSF calculation that best fulfilled the above criteria described the grains' interaction and consequently the intergranular stress generated between elastically anisotropic crystallites. These models were then used to determine residual stress, lattice parameter $\left(a_{0}\right)$ and stacking fault probability $(\rho)$.

In our experiment, the austenite stainless steel sample was subjected to controlled tension $\left(\Sigma_{11}=50,180,300,180,50\right.$ and $0 \mathrm{MPa}$ ) during loading and unloading in a tensile machine placed in a diffractometer. The applied values of stress $\Sigma_{11}$ were below the yield stress (determining the linear range of deformation) of the AISI316L sample, which was over $380 \mathrm{MPa}$. In the case of the MGIXD method, the $\langle\varepsilon(\phi, \psi)\rangle_{\{h k l\}}^{\mathrm{rel}}$ relative strains were measured for $\alpha=20^{\circ}$ (corresponding to a penetration depth of $\tau=3.5 \mu \mathrm{m}$ ), while in the standard method, the $\chi$ geometry with the 311 reflection was applied (mean penetration depth $\tau=7.7 \mu \mathrm{m}$ ). The surface roughness of the sample did not influence either of our measurements,

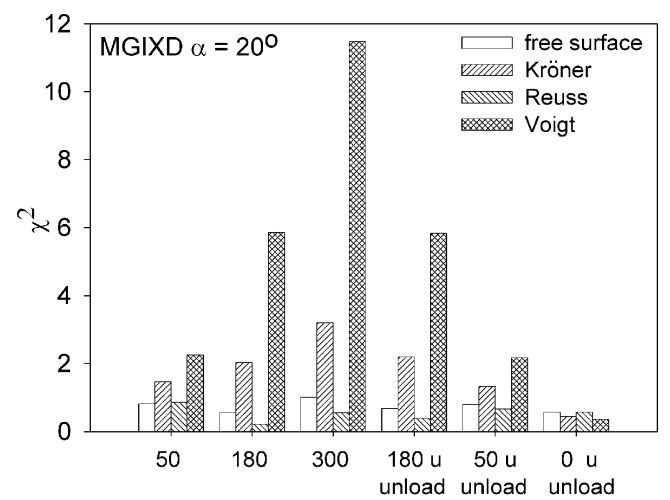

Figure 9

applied stress (MPa)

Comparison of the values of $\chi^{2}$ obtained from analysis of the MGIXD measurements performed during uniaxial loading and unloading of the austenitic sample. The results for the four grain interaction models are shown. 
because the penetration depth $\tau$ was more than 30 times greater than the mean roughness parameter $R_{\mathrm{a}}=0.11 \mu \mathrm{m}$, as measured by a Veeco WYKO NT 930 optical profilometer.
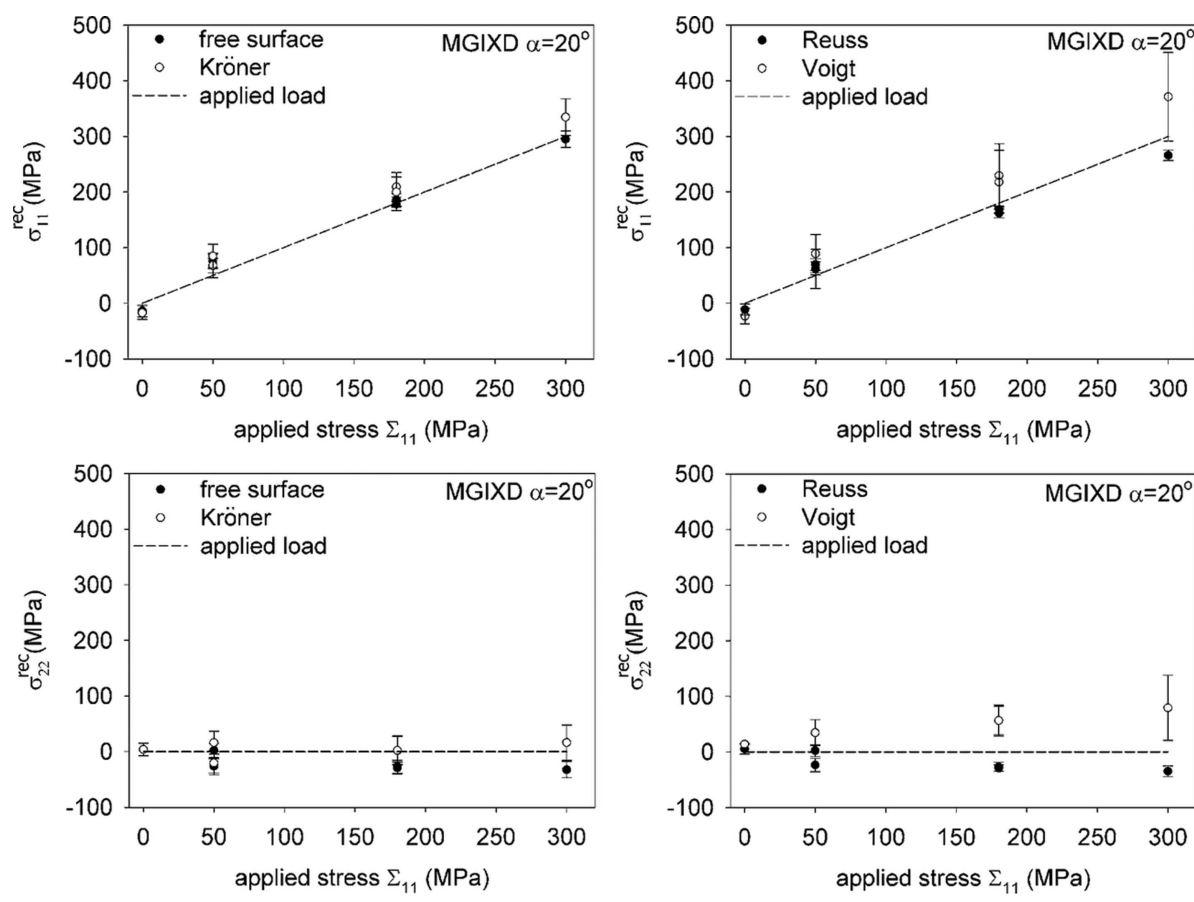

(a)
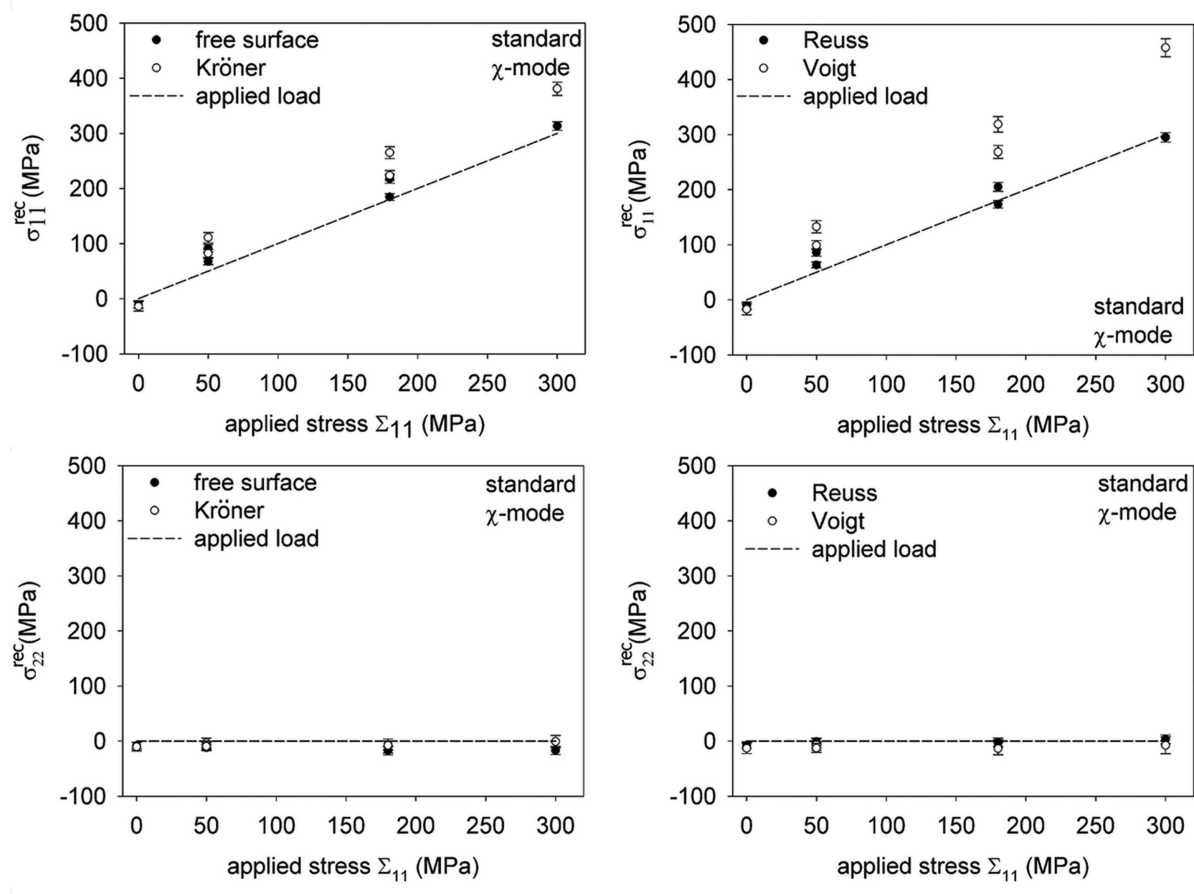

(b)

Figure 10

Comparison of the recalculated values of stress $\sigma_{11}^{\text {rec }}$ and $\sigma_{22}^{\text {rec }}$ (points) with applied stress $\Sigma_{11} \neq 0$ and $\Sigma_{22}=0 \mathrm{MPa}$ (dashed line, for which $\sigma_{i i}^{\text {rec }}=\Sigma_{i i}$ is assumed). Results of loading and unloading of the sample are shown versus $\Sigma_{11}$ (point $\Sigma_{11}=0 \mathrm{MPa}$ corresponds to the state after complete unloading) for different grain interaction models. The measurements were performed using $(a)$ the MGIXD method and $(b)$ the standard method ( $\chi$ mode with the 311 reflection).
The experimental relative lattice strain $\langle\varepsilon(\phi, \psi)\rangle_{\{h k l\}}^{\mathrm{rel}}$ versus $\sin ^{2} \psi$ plots determined according to equation (11) and the curves fitted using equation (12) are presented in Fig. 8. The resutls corresponding to the four grain interaction models and different applied loads are shown. As was demonstrated in the previous section (see $\S 3$ ), the nonlinearity of the $\sin ^{2} \psi$ plots in Fig. 8 is associated with strong elastic anisotropy in the sample.

From the $\sin ^{2} \psi$ plots in Fig. 8, it can be seen that the Reuss, Kröner and free-surface models came very close to the experimental values. The linear dependence of the lattice strains versus $\sin ^{2} \psi$ predicted by the Voigt model did not agree with the lattice strains measured for the austenite stainless steel with strong elastic anisotropy.

A quantitative comparison of the fitting quality is given by the $\chi^{2}$ parameter [equation (13)], the values for which are compared in Fig. 9 for all applied loads and for the four tested models. For perfectly predicted values of the XSFs, the $\chi^{2}$ parameter should depend only on experimental uncertainties and should not increase even for large values of applied stress (an increase/decrease in $\chi^{2}$ indicates an increase/decrease in the differences between the theoretical and experimental values). In this case, the measured relative lattice strain caused by applied stress changes by the same value as the theoretically predicted one; consequently their differences do not change [i.e. the difference $\left\langle\varepsilon\left(\phi_{n}, \psi_{n}\right)\right\rangle_{\{h k l\}}^{\exp }-\left\langle\varepsilon\left(\phi_{n}, \psi_{n}\right)\right\rangle_{\{h k l\}}^{\mathrm{cal}}$ in equation (13) is the same for various applied loads]. However, if the XSF values are incorrectly calculated by the model, the difference between the theoretical and the experimental lattice strains [i.e. $\left\langle\varepsilon\left(\phi_{n}, \psi_{n}\right)\right\rangle_{\{h k l\}}^{\exp }-$ $\left.\left\langle\varepsilon\left(\phi_{n}, \psi_{n}\right)\right\rangle_{\{h k l\}}^{\mathrm{cal}}\right]$ and the value of $\chi^{2}$ should significantly increase with an increase in the applied load. Comparing the values of $\chi^{2}$ and their dependence on the applied load, it can be concluded that the Reuss and freesurface models correctly describe the elastic anisotropy of the XSFs (Fig. 9). For these models, the value of $\chi^{2}$ is small and remains almost constant for all applied external stress. In contrast, the $\chi^{2}$ values obtained for the Kröner 
Table 2

Values of stress recalculated from relative lattice strains for the AISI316L sample under a load $\Sigma_{11}=300 \mathrm{MPa}$ using different XSF models.

Results taking experimental texture (Fig. 4a) into account are compared with assumptions of random lattice orientations. Values in parentheses are uncertainties on the least significant digits.

\begin{tabular}{|c|c|c|c|c|c|c|}
\hline \multirow[b]{2}{*}{ Model } & \multicolumn{3}{|c|}{ MGIXD method } & \multicolumn{3}{|c|}{ Standard method } \\
\hline & $\sigma_{11}^{\text {rec }}(\mathrm{MPa})$ & $\sigma_{22}^{\mathrm{rec}}(\mathrm{MPa})$ & $\chi^{2}$ & $\sigma_{11}^{\mathrm{rec}}(\mathrm{MPa})$ & $\sigma_{22}^{\mathrm{rec}}(\mathrm{MPa})$ & $\chi^{2}$ \\
\hline \multicolumn{7}{|c|}{ With texture from Fig. $4(a)$} \\
\hline Reuss & $266(10)$ & $-35(10)$ & 0.6 & $295(9)$ & $3(8)$ & 4.0 \\
\hline Free surface & $295(15)$ & $-33(14)$ & 1.0 & $314(8)$ & $-17(8)$ & 3.1 \\
\hline Kröner & $335(33)$ & $16(32)$ & 3.2 & $381(12)$ & $-1(11)$ & 4.8 \\
\hline Voigt & $371(80)$ & $58(76)$ & 11.5 & $458(16)$ & $-8(16)$ & 6.0 \\
\hline \multicolumn{7}{|c|}{ Random orientations } \\
\hline Reuss & $263(18)$ & $-32(18)$ & 1.9 & $268(10)$ & $-10(10)$ & 6.0 \\
\hline Free surface & $288(23)$ & $-25(23)$ & 2.5 & $291(9)$ & $-21(9)$ & 4.9 \\
\hline Kröner & $322(33)$ & $15(33)$ & 3.5 & 349 (13) & $-14(13)$ & 6.0 \\
\hline Voigt & 349 (76) & $53(76)$ & 11.5 & 428 (16) & $-16(16)$ & 6.0 \\
\hline
\end{tabular}

and Voigt models were large and increased with applied stress. This effect was especially significant in the case of the Voigt model, in which the linear dependence of the lattice strains versus $\sin ^{2} \psi$ was predicted by the model.

To confirm whether the second criterion of the model verification was fulfilled, the stress $\left(\sigma_{11}^{\text {rec }}\right)$ in the direction of the load and the stress $\left(\sigma_{22}^{\text {rec }}\right)$ perpendicular to the load were determined from the measured relative lattice strains using different XSF models. In Fig. 10 the recalculated values of stress $\left(\sigma_{11}^{\text {rec }}\right.$ and $\left.\sigma_{22}^{\text {rec }}\right)$ versus applied $\Sigma_{11}$ stress are presented for the loaded and unloaded samples. The results of measurements applying the MGIXD method are shown in Fig. 10(a), while the results for the standard geometry are presented in Fig. 10(b). It can be seen that the loading and unloading processes are completely reversible (almost the same recalculated values of stress were obtained for identical applied stress during loading and unloading). This confirms that the measurements were performed within the elastic range of deformation. Moreover, the $\sigma_{11}^{\text {rec }}$ stress measured after complete sample unloading is very close to a value of zero (within the uncertainty range, $c f$. the results for $\Sigma_{11}=0 \mathrm{MPa}$ in Fig. 10). The stress state in the sample was successfully determined from diffraction data, i.e. the measured value of the stress $\sigma_{11}^{\text {rec }}$ approaches the applied stress $\Sigma_{11}$, while $\sigma_{22}^{\text {rec }}$ is always close to zero for both the standard and MGIXD methods in the case of the Reuss and free-surface models. The $\sigma_{11}^{\text {rec }}$ stress calculated using the Kröner method deviates slightly from the $\Sigma_{11}$ value, and a large disagreement between the recalculated $\left(\sigma_{11}^{\text {rec }}\right)$ and applied values of stress was found when the XSFs were calculated according to the Voigt approach. It can also be seen that in the case of the incorrect model (especially for the Voigt model) a large deviation between the applied and measured values of stress occurs for the standard stress measurements (Fig. 10b), while in the case of MGIDX this deviation is smaller, but a large uncertainty of the determined values was obtained (Fig. 10a). The analysis of the recalculated values of stress performed above confirms the conclusions based on comparison of the $\chi^{2}$ values obtained by using different methods.
Finally, the influence of the texture function on the determined values of stress was studied. For this purpose, the $\sigma_{11}^{\text {rec }}$ and $\sigma_{22}^{\text {rec }}$ determined using the XSFs predicted by the different models, taking into account the experimental texture (Fig. 4a) or assuming random texture ( $c f$. Table 2), were compared. It was found that the influence of texture on the recalculated values of stress is not significant (much smaller than the experimental uncertainties) and that the choice of deformation model plays a key role in stress analysis performed for the studied austenitic stainless steel (AISI316L). It can also be seen that the uncertainties of the determined stress were significantly smaller when the texture function was used to calculate XSFs.

Summarizing the presented results concerning the quality of strain fitting, as well as the values of recalculated stress, it appears that the Reuss and free-surface models can be considered the best approximations for XSF calculation in the case of elastically anisotropic materials, such as the austenitic stainless steel studied in the present paper.

\section{Measurements of residual stress}

Samples with significant surface residual stress but not subjected to external load were investigated in this part of the study. For simplicity, only samples with negligible $\sigma_{13}^{\text {res }}$ and $\sigma_{23}^{\text {res }}$ residual stress components were selected. The XSFs were calculated from the single-crystal elastic constants given in Table 1 and using the textures presented in Fig. 4.

The first studied sample was a cylindrical sample of highpurity tungsten [the sample for which crystallographic texture is presented in Fig. 4(b)]. To generate stress in the surface layer, a cross section of the bar was manually polished (2000 grit paper, nondirectional polishing), resulting in a roughness of $R_{\mathrm{a}}=0.16 \mu \mathrm{m}$.

The influence of stacking faults on stress analysis was investigated for austenitic hot-rolled stainless steel (AISI316L) samples, the surfaces of which were subjected to various mechanical treatments. The surface of the first sample was ground in one direction with a workpiece speed of $1 \mathrm{~m} \mathrm{~min}^{-1}$ and depth of cut of $1 \mu \mathrm{m}$. The second sample was manually polished with 2000 grit paper in two perpendicular directions in order to generate stress in the surface layer. The average roughness of the surface was $R_{\mathrm{a}}=0.3 \mu \mathrm{m}$ for the ground and $R_{\mathrm{a}}=0.13 \mu \mathrm{m}$ for the mechanically polished sample.

It should be stated that the surface roughness influences the results of stress measurements, changing the peak position and decreasing the intensity of the measured diffraction peak (Marques et al., 2006). To avoid this problem, it was recommended in previous studies that stress should be measured for a penetration depth $\tau$ (or average penetration depth) greater than twice the $R_{\mathrm{a}}$ value in the case of standard methods (Hauk, 1997) or greater than $R_{\mathrm{a}}$ in the case of grazing-incidence measurements (Marques et al., 2006). In this study, these criteria were fulfilled for all performed measurements. The residual stress components were measured using MGIXD for minimum penetration depths equal to $0.24 \mu \mathrm{m}$ for mechani- 
Table 3

Values of stress and the $a_{0}$ lattice parameter determined in a polished W sample using the MGIXD (different $\alpha$ angles) and standard methods ( $\omega$ and $\chi$ modes).

All four grain interaction models led to the same results. Penetration depth was calculated as an average over the $\sin ^{2} \psi$ range

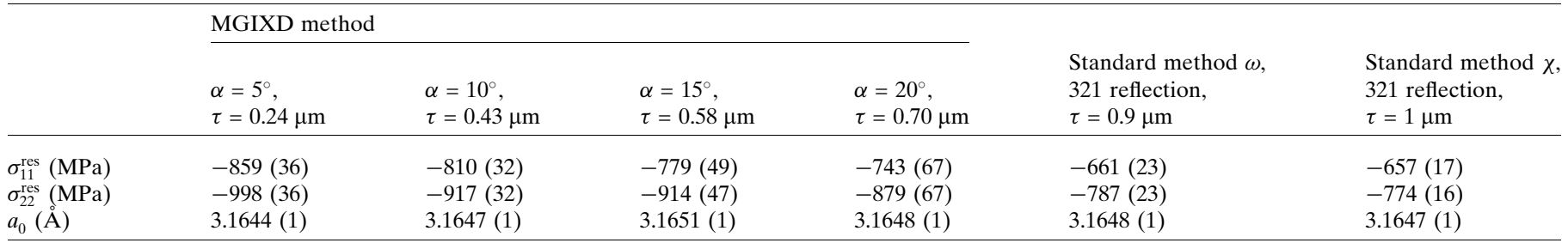

cally polished tungsten and $0.55 \mu \mathrm{m}$ for ground and $1.41 \mu \mathrm{m}$ for mechanically polished austenite. In the standard method, the mean penetration over the measured range of $\sin ^{2} \psi$ was $6.13 \mu \mathrm{m}$ for ground austenitic steel and $0.9 \mu \mathrm{m}$ for polished tungsten.

\subsection{Mechanically polished tungsten sample}

First, an elastically isotropic sample ( $A=1$; Table 1$)$ of highpurity tungsten (W) was investigated with $\mathrm{Cu} K \alpha$ radiation on a PANalytical X'Pert MRD diffractometer. A parallel beam configuration was used, with a Göbel mirror for incident beam optics and a parallel plate collimator (Söller collimator) for reflected beam optics. The MGIXD method for different incident $\alpha$ angles and the standard method ( $\omega$ and $\chi$ geometries with the 321 reflection) were used to measure lattice strains. In order to calculate stress, a fitting procedure based

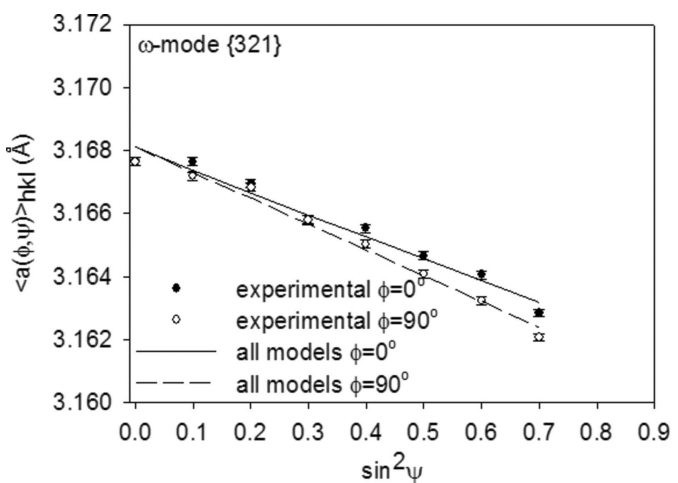

(a)

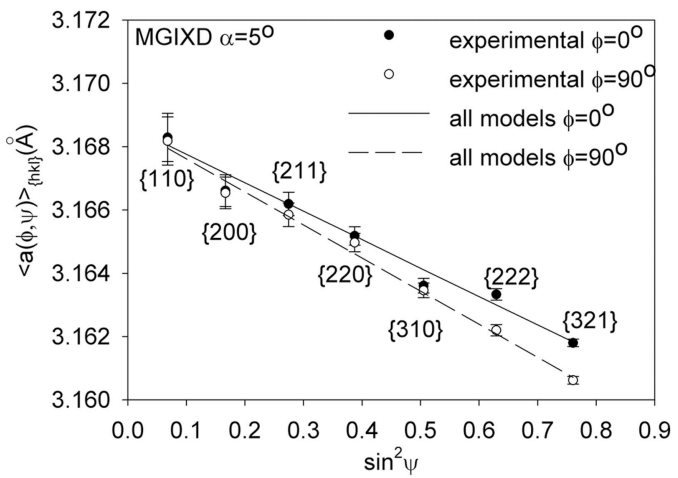

$(c)$ on equation (6) was applied, and two components, $\sigma_{11}^{\text {res }} \simeq \sigma_{22}^{\text {res }}$, of the biaxial stress tensor as well as the $a_{0}$ strain-free lattice parameter were determined (Table 3 ). In the analysis, the XSFs were calculated with the Kröner model from the singlecrystal elastic constants given in Table 1, using the ODF shown in Fig. 4(b). However, it should be mentioned that, in the case of the isotropic W sample, neither the choice of model nor the crystallographic texture influenced the theoretical values of the XSFs. The $\langle a(\phi, \psi)\rangle_{\{h k l\}}$ versus $\sin ^{2} \psi$ plots for the standard methods ( $\omega$ and $\chi$ modes) and for the MGIXD method (with different incident angles $\alpha$ ) are shown in Fig. 11. It should be noted that the results obtained with the four XSF models are linear and that the fitted plots overlap each other.

Whether MGIXD or the standard methods are used, the values of determined stress for any of the chosen grain interaction models do not differ significantly. In the case of the

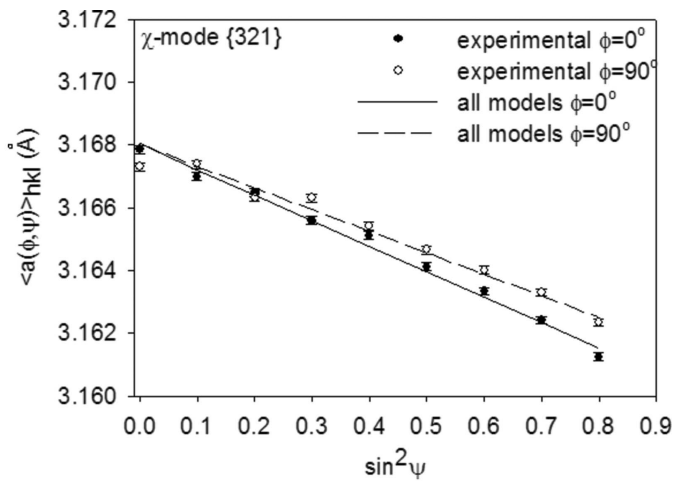

(b)

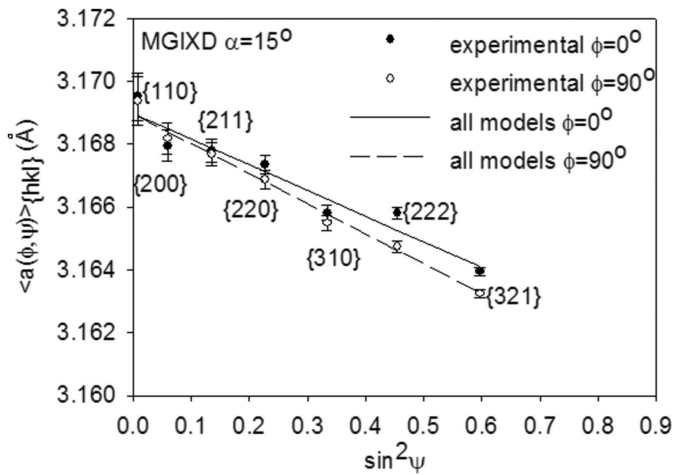

(d)

Figure 11

Experimental points and theoretical $\langle a(\phi, \psi)\rangle_{\{h k l\}}$ versus $\sin ^{2} \psi$ plots for a polished tungsten sample. Measurements presented for the standard $\omega$ geometry $(a)$, the standard $\chi$ geometry $(b)$, and the MGIXD method with incident angles $\alpha=5^{\circ}(c)$ and $\alpha=15^{\circ}(d)$ [uncertainty of peak position $\delta(2 \theta)=0.01^{\circ}$ was assumed]. 
elastically isotropic tungsten sample, the $\langle a(\phi, \psi)\rangle_{\{h k l\}}$ versus $\sin ^{2} \psi$ plots are linear for all considered models (Fig. 11). This is certainly due to the perfect elastic isotropy of $\mathrm{W}$ crystals and consequently equal values of XSFs for all reflections.

The stress in the mechanically polished $\mathrm{W}$ is compressive and biaxial and approximately fulfils the relationship $\sigma_{11}^{\text {res }} \simeq \sigma_{22}^{\text {res }}($ see Table 3$)$. The stress value determined by the MGIXD method slowly decreases with penetration depth and agrees perfectly with the results of both standard methods. The values of stress obtained with the standard methods were calculated for an average value of penetration depth for all $\psi$ inclination angles. A small curvature of the $\sin ^{2} \psi$ plots due to a stress gradient is observed in Fig. 11 for the standard $\chi$ and $\omega$ geometries. Indeed, it can be seen that the slope of the $\sin ^{2} \psi$ plots is smaller at the higher penetration depths (low $\psi$ values) than at the shallower depths (high $\psi$ values). The measured value of the strain-free lattice parameter $a_{0}$ does not change significantly with depth and is nearly constant for the different models used in the analysis (see Table 3).

It can be concluded that, although the results of the MGIXD method exhibit greater uncertainty (especially due to small values of the $2 \theta$ angle for low $h \mathrm{kl}$ reflections) in comparison with the standard methods, one indisputable advantage of grazing-incidence geometry is its capacity to perform stress measurement at strictly defined depths. In the sample studied above, which exhibited a small stress gradient, the stress evolution versus penetration depth was determined using MGIXD and reasonable values were obtained (the results are comparable to those obtained from standard methods). However, in the case of very strong gradients, only grazing methods enable reliable stress measurement, whereas the interpretation of the standard method requires additional assumptions concerning stress evolution under the surface.

\subsection{Mechanically treated austenitic sample}

The presented methodology of stress and stacking fault analysis requires knowledge of the XSFs for the anisotropic material. Different models for

Figure 12
XSF calculation had already been verified by measuring lattice strains during the tensile test $(\S 4)$. It was found that the values of stress recalculated from the measured relative lattice strains agreed with the values of applied stress when the free-surface and Reuss models were used for calculation of the XSFs. Therefore, these models were also used in the analysis of residual stress performed for the austenitic sample. The XSFs were calculated from single-crystal elastic constants (Table 1),
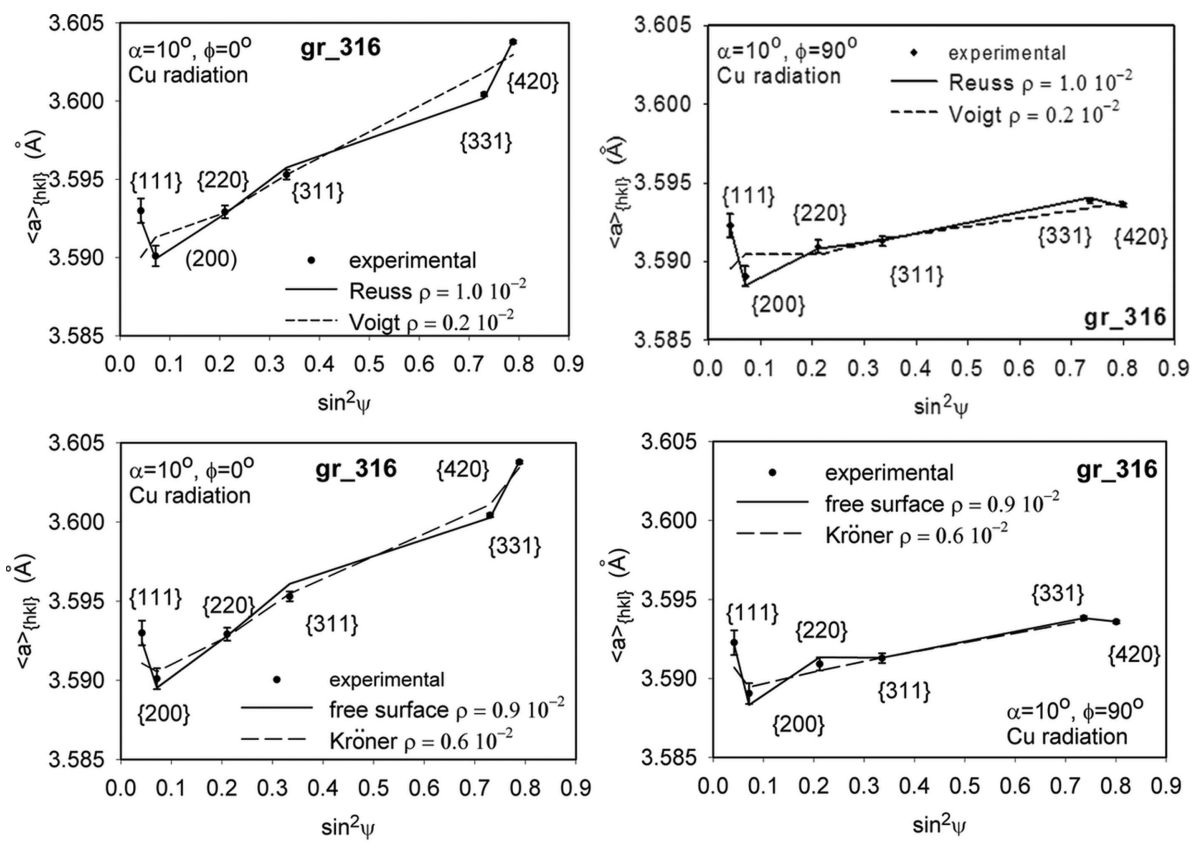

(a)
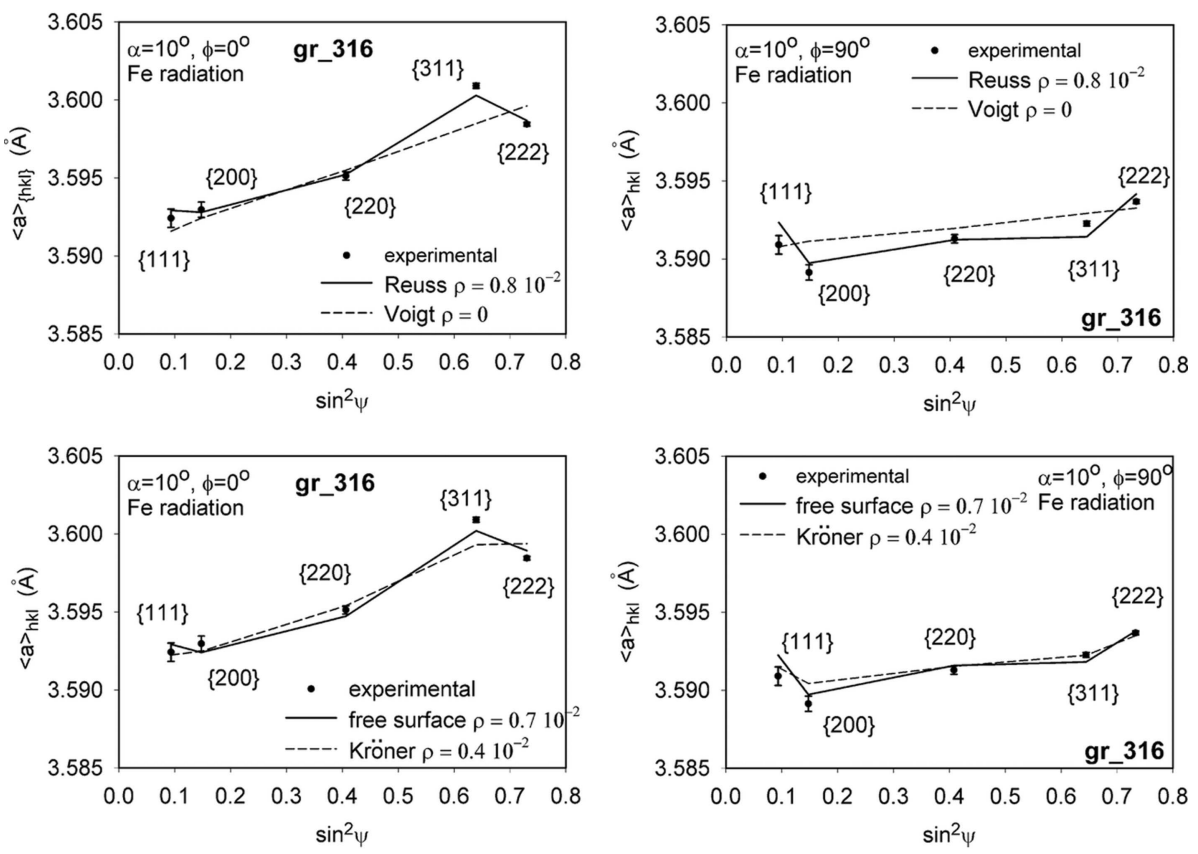

(b)

The $\langle a(\phi, \psi)\rangle_{\{h k l\}}$ lattice parameters fitted to the experimental points for the AISI316L sample using equation (10) and different types of XSF models. (a) $\mathrm{Cu} K \alpha$ radiation and (b) $\mathrm{Fe} K \alpha$ radiation were used, and the plots for $\phi=0^{\circ}$ and for $\phi=90^{\circ}$ are shown. The results of the fitting are listed in Table 3; the uncertainty of the peak position is equal to $\delta(2 \theta)=0.01^{\circ}$. 
taking into account experimentally determined crystallographic texture (Fig. 4a).

In this study, the influence of stacking faults on the determined $\langle a(\phi, \psi)\rangle_{\{h k l\}}$ parameters was taken into account in the stress analysis. An interpretation of the grazingincidence experiment based on equation (10) was performed and the probability of stacking faults was estimated.

5.2.1. Ground surface of austenitic stainless steel. In order to increase the available range of penetration depth, measurements were performed (for $\phi=0^{\circ}$ and $\phi=$ $90^{\circ}$ ) using three wavelengths of X-ray radiation $(\mathrm{CuK} \alpha$ on a PANalytical X'Pert MRD, Fe $K \alpha$ and Mn $K \alpha$ on Seifert PTS MZ VI diffractometers) for which the coef-

ficients of absorption in the studied ground AISI316L steel were different. In the case of the MGIXD method, $\mathrm{Cu} K \alpha$ and Fe $K \alpha$ radiation were applied, while the standard measurement ( $\omega$ geometry for the 311 reflection) was done with $\mathrm{Mn} K \alpha$ radiation. Examples of stress analysis results for ground AISI316L steel are shown in Table 4 and in Fig. 12 (the sample frame is defined by the axes $x_{1}\left\|\mathrm{TD}, x_{2}\right\| \mathrm{RD}$ and $\left.x_{3} \| \mathrm{ND}\right)$. It can be concluded that the determined values of stress change only insignificantly when the influence of stacking faults is taken into account in the fitting procedure $(\rho \neq 0$ in Table 4$)$. As expected, the presence of stacking faults mostly affects the magnitude of the nonlinearities but does not significantly modify the slope of the $\langle a(\phi, \psi)\rangle_{\{h k l\}}$ versus $\sin ^{2} \psi$ plot.

Different types of X-ray stress factors were used to determine stress from the experimental data (Table 4 and Fig. 12). The best fit quality (smallest values of $\chi^{2}$ and small degree of uncertainty of the determined stress) was found for the freesurface model; only slightly poorer results were obtained for the Reuss model. In the case of the Kröner and Voigt models, the value of the $\chi^{2}$ parameter significantly increased; moreover, the fitted plots did not match the experimental points even when an additional $\rho$ parameter was used in the fitting procedure (Fig. 12).

Table 4

Figure 13 models are shown.
Comparison of macrostress $\left(\sigma_{i i}^{\text {res }}\right)$, stacking fault probability $(\rho)$ and the $\chi^{2}$ parameter determined for ground AISI316L austenite using different XSF models.

\begin{tabular}{|c|c|c|c|c|c|c|}
\hline \multirow{2}{*}{$\begin{array}{l}\text { Sample and X-ray } \\
\text { radiation; } \\
\text { incident angle; } \\
\text { penetration depth }\end{array}$} & \multirow[b]{2}{*}{$\begin{array}{l}\text { Analysis of } \\
\text { stacking } \\
\text { fault effect }\end{array}$} & \multirow[b]{2}{*}{$\begin{array}{l}\sigma_{i i}^{\text {res }}(\mathrm{MPa}) \\
\rho\left(10^{-2}\right) \\
\chi^{2}\end{array}$} & \multicolumn{4}{|c|}{ Results of fitting assuming $\sigma_{33}^{\text {res }}=0$} \\
\hline & & & Voigt & Kröner & Free surface & Reuss \\
\hline \multirow{7}{*}{ 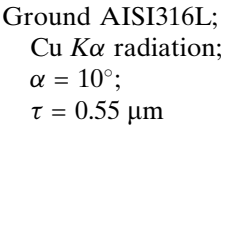 } & \multirow[t]{3}{*}{ No } & $\sigma_{11}^{\text {res }}$ & $824(81)$ & $707(41)$ & $603(32)$ & $545(34)$ \\
\hline & & $\sigma_{22}^{\text {res }}$ & $259(81)$ & $211(41)$ & $143(31)$ & $144(34)$ \\
\hline & & $\chi^{2}$ & 30 & 10 & & 12 \\
\hline & \multirow[t]{4}{*}{ Yes } & $\sigma_{11}^{\text {res }}$ & $821(98)$ & $707(35)$ & $608(13)$ & $554(13)$ \\
\hline & & $\sigma_{22}^{\text {res }}$ & $256(98)$ & $210(35)$ & 149 (13) & 154 (13) \\
\hline & & $\rho$ & $0.2(6)$ & $0.6(3)$ & $0.9(1)$ & $1.0(1)$ \\
\hline & & $\chi^{2}$ & 33 & 8 & 2 & 2 \\
\hline \multirow{7}{*}{$\begin{array}{l}\text { Ground AISI316L; } \\
\text { Fe } K \alpha \text { radiation; } \\
\quad \alpha=10^{\circ} \\
\tau=2.6 \mu \mathrm{m}\end{array}$} & \multirow[t]{3}{*}{ No } & $\sigma_{11}^{\text {res }}$ & $610(150)$ & $563(88)$ & $490(59)$ & $452(60)$ \\
\hline & & $\sigma_{22}^{\text {res }}$ & $190(150)$ & $120(88)$ & $39(59)$ & $31(60)$ \\
\hline & & $\chi^{2}$ & 55 & 28 & & 22 \\
\hline & \multirow[t]{4}{*}{ Yes } & $\sigma_{11}^{\text {res }}$ & $610(170)$ & $551(91)$ & $491(43)$ & 459 (45) \\
\hline & & $\sigma_{22}^{\text {res }}$ & $190(170)$ & $110(91)$ & $43(43)$ & $39(45)$ \\
\hline & & $\rho$ & $0.4(8)$ & $0.4(5)$ & $0.7(3)$ & $0.8(4)$ \\
\hline & & $\chi^{2}$ & 64 & 29 & 9 & 12 \\
\hline
\end{tabular}

The values of determined stress $\left(\sigma_{11}^{\text {res }}\right.$ and $\left.\sigma_{22}^{\text {res }}\right)$ and the strain-free lattice parameter $\left(a_{0}\right)$, as well as the probability of finding stacking faults $(\rho)$, as a function of penetration depth $(\tau)$ are shown in Fig. 13. It should be noted that good consistency was obtained for all measured quantities within the depth ranges available for $\mathrm{Cu} K \alpha$ and $\mathrm{Fe} K \alpha$ radiation. Also, stress measured using the standard method with $\mathrm{Mn} K \alpha$
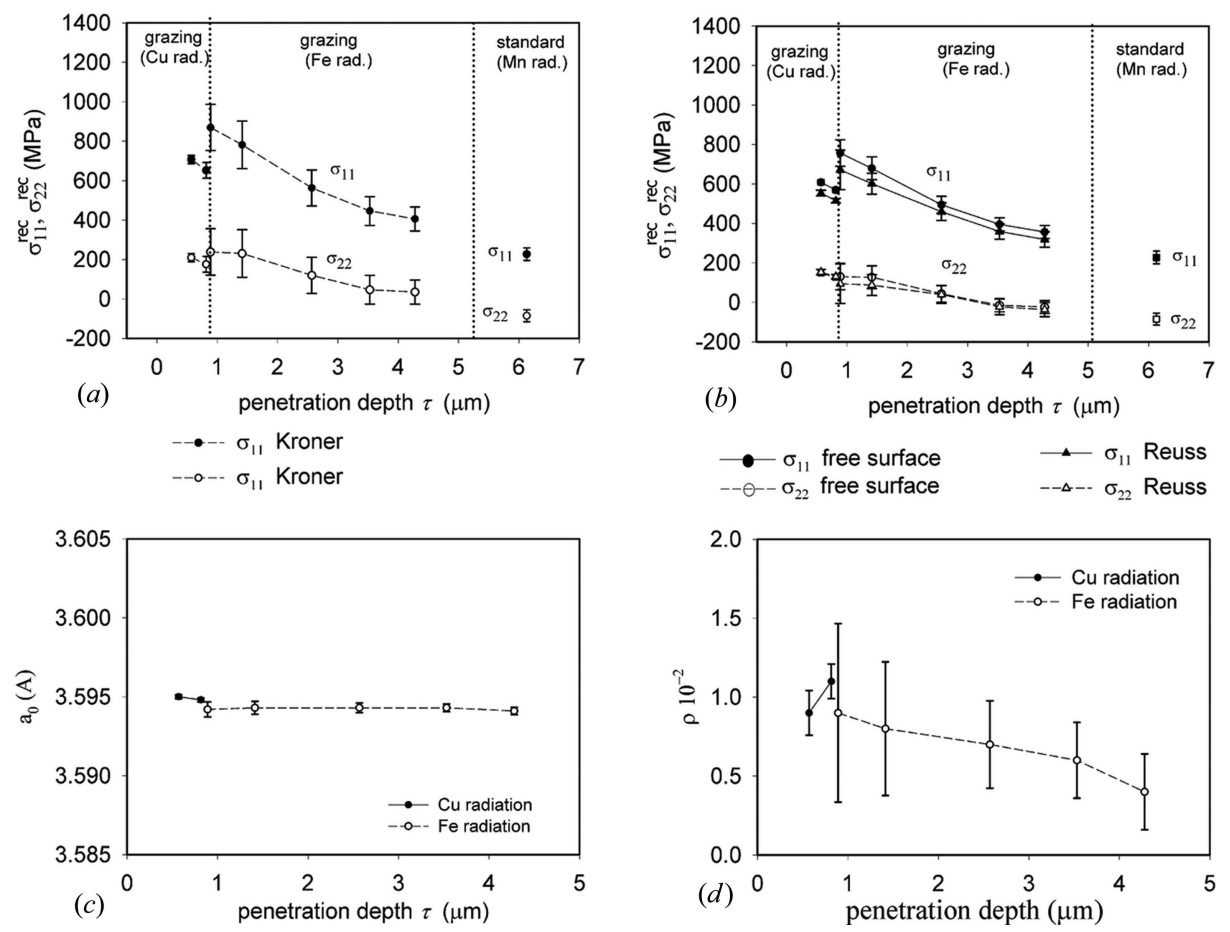

Stress $\left(\sigma_{11}^{\text {res }}\right.$ and $\left.\sigma_{22}^{\text {res }}\right)$, lattice parameter $\left(a_{0}\right)$ and the probability of finding stacking faults $(\rho)$ plotted versus penetration depth $\tau$ for the ground sample AISI316L. The free-surface model was used to calculate the XSFs. For comparison, the values of stress obtained with the $(a)$ Kröner and $(b)$ Reuss 
radiation (mean value $\tau \simeq 6 \mu \mathrm{m}$ for the 311 reflection) confirmed the decreasing trend of stress versus penetration depth dependences determined using the MGIXD method. As shown in Fig. 13 (results of the free-surface model), the value of the measured stressfree parameter $a_{0}=3.5945$ (10) $\AA$ is almost constant versus the penetration depth, while the probability of finding stacking faults $(\rho)$ progressively decreases with the depth below the sample surface. It should be emphasized that when $\mathrm{Cu} K \alpha$ radiation was used the value of $\rho$ was determined close to the sample surface with reasonable uncertainty; however, when measured deeper with $\mathrm{Fe} K \alpha$ radiation, great uncertainty of the $\rho$ value was obtained. In general, the uncertainties of all quantities determined using $\mathrm{Fe} K \alpha$ radiation are larger than those measured with $\mathrm{Cu} K \alpha$ radiation. This is because a narrower range of $\sin ^{2} \psi$ and a smaller number of reflections are available for $\mathrm{Fe} K \alpha$ than for $\mathrm{Cu} K \alpha$ radiation (cf. Figs. $12 a$ and $12 b$ ).

5.2.2. Polished surface of austenitic stainless steel. Stress measurements for polished AISI316L steel were performed using Fe $K \alpha$ radiation (Seifert PTS

MZ VI) for $\phi=0^{\circ}$ and $\phi=90^{\circ}$. Examples of the results are shown in Table 5 (the sample frame was defined in the same way as for the ground sample). Similarly to the ground sample, it was found that stacking faults taken into account in the stress analysis do not significantly change the stress values determined using the XSFs calculated by the Kröner, free-surface and Reuss models (Table 5). For these models, a relatively good fit quality was obtained, characterized by low values of $\chi^{2}$. Only in the case of the Voigt model was the value of the $\chi^{2}$ parameter significantly high; consequently this model cannot be used to analyse the stress state in a polished sample. However, despite the low $\chi^{2}$ values, the values of stress determined using the Kröner model did not agree with those obtained from the free-surface and Reuss models (Table 5). This means that the criterion of fit quality cannot be used to select the correct approach and the model must be verified using the in situ tensile test,
Table 5

Comparison of macrostress $\left(\sigma_{i i}^{\text {res }}\right)$, stacking fault probability $(\rho)$ and the $\chi^{2}$ parameter determined for polished AISI316L austenite using different XSF models.

\begin{tabular}{|c|c|c|c|c|c|c|}
\hline \multirow[b]{2}{*}{$\begin{array}{l}\text { Sample and X-ray } \\
\text { radiation; } \\
\text { incident angle; } \\
\text { penetration depth }\end{array}$} & \multirow[b]{2}{*}{$\begin{array}{l}\text { Analysis of } \\
\text { stacking } \\
\text { fault effect }\end{array}$} & \multirow[b]{2}{*}{$\begin{array}{l}\sigma_{i i}^{\text {res }}(\mathrm{MPa}), \\
\rho\left(10^{-2}\right), \\
\chi^{2}\end{array}$} & \\
\hline & & & Voigt & Kröner & Free surface & Reuss \\
\hline $\begin{array}{l}\text { Polished AISI316L, } \\
\text { Fe } K \alpha \text { radiation; } \\
\begin{array}{l}\alpha=10^{\circ} \\
\tau=2.6 \mu \mathrm{m}\end{array}\end{array}$ & No & $\begin{array}{l}\sigma_{11}^{\text {res }} \\
\sigma_{22}^{\text {res }} \\
\chi^{2} \\
\sigma_{11}^{\text {res }} \\
\sigma_{22}^{\text {res }} \\
\rho \\
\chi^{2}\end{array}$ & $\begin{array}{l}-270(190) \\
-580(180) \\
84 \\
-420(120) \\
-720(110) \\
1.9(5) \\
27\end{array}$ & $\begin{array}{l}-280(120) \\
-590(110) \\
44 \\
-316(57) \\
-628(54) \\
1.4(3) \\
9.8\end{array}$ & $\begin{array}{l}-215(78) \\
-533(74) \\
29 \\
-210(53) \\
-528(51) \\
0.9(3) \\
13\end{array}$ & $\begin{array}{l}-219(67) \\
-507(64) \\
24 \\
-210(48) \\
-498(46) \\
0.8(3) \\
12\end{array}$ \\
\hline
\end{tabular}

as was done in this study. From the previous results, it was concluded that the free-surface and Reuss models of XSF calculation agreed with the experimental results obtained from the tensile test. Therefore, the values of stress determined for the polished austenitic sample with these two models (whose results are not significantly different) can be assumed to be the correct ones (Fig. 14b).

It was found that the stress in the polished sample is compressive and almost constant for the studied depth, and that the $\sigma_{11}^{\text {res }}$ and $\sigma_{22}^{\text {res }}$ components differ from each other. The value of the stress-free parameter, $a_{0}=3.5933$ (20) $\AA$, does not
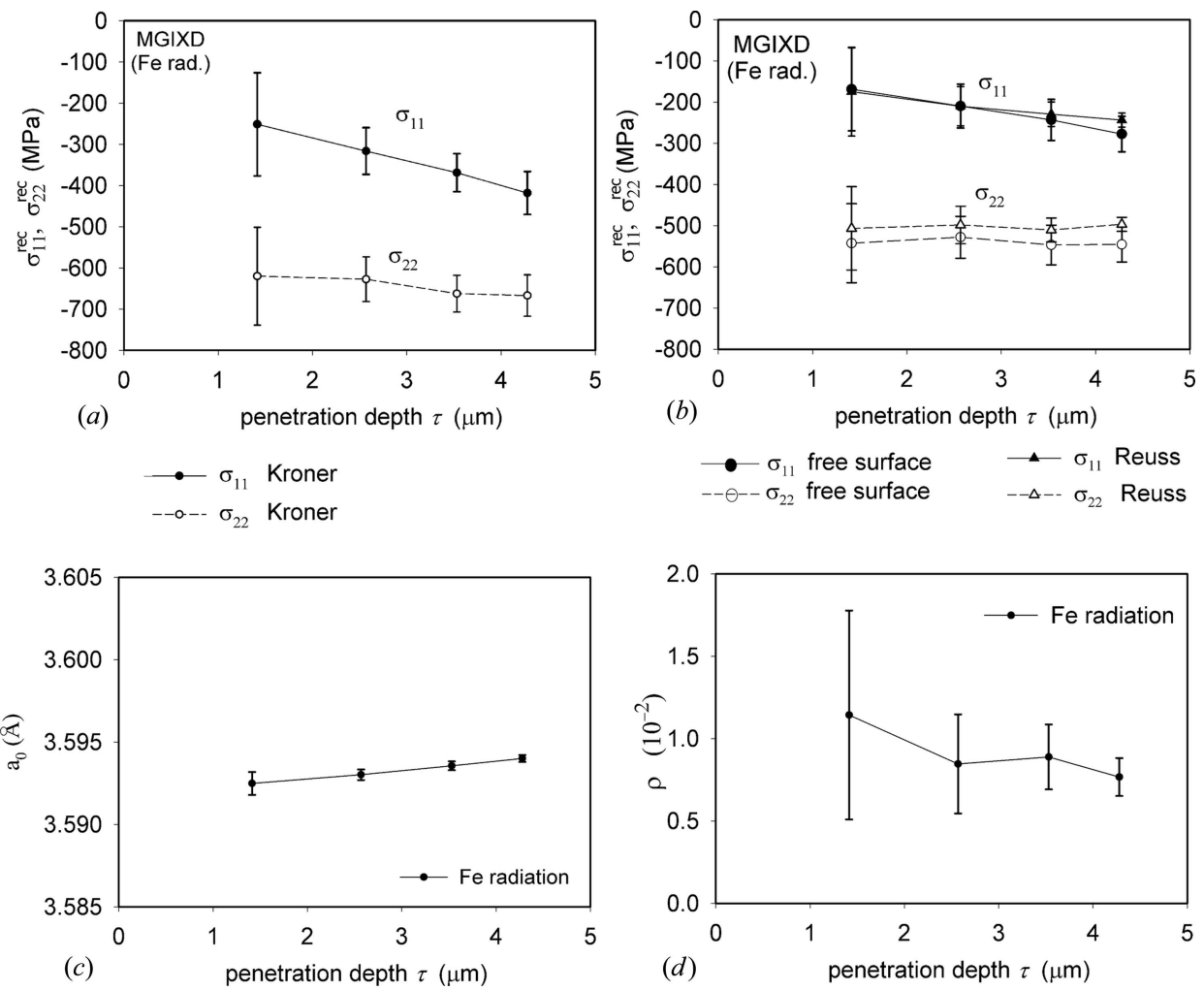

Figure 14

Stress $\left(\sigma_{11}^{\text {res }}\right.$ and $\left.\sigma_{22}^{\text {res }}\right)$, lattice parameter $\left(a_{0}\right)$ and the probability of finding stacking faults $(\rho)$ determined from equation (10), plotted versus penetration depth $\tau$ for the polished AISI316L sample. The freesurface model was used to calculate the XSFs. For comparison, the values of stress obtained with the $(a)$ Kröner and (b) Reuss models are shown. 
change significantly versus penetration depth and its value is close to that determined for the ground sample (Fig. 14c).

As shown in Fig. 14(d), the probability of stacking faults $(\rho)$ in polished austenite is similar to that determined for the ground sample. Similar values of $\rho$ were found from the fitting procedure [based on equation (10)] when the XSFs were calculated using the free-surface and Reuss methods (Fig. 15). For these approaches, the values of the $\chi^{2}$ parameter are rather low (Table 5), but the uncertainty of $\rho$ is relatively large. In the case of XSFs calculated by the Kröner model, a higher value of the $\rho$ parameter, with very similar uncertainty, was found. Again, the poorest results and a very poor fit were obtained when using the Voigt model (Table 5).

The MGIXD method was used to investigate the influence of stacking faults on the determined values of $\langle a(\phi, \psi)\rangle_{\{h k l\}}$ in polished austenite. As shown in Fig. 16, the presence of stacking faults again affected the nonlinearities of the $\langle a(\phi, \psi)\rangle_{\{h k l\}}$ versus $\sin ^{2} \psi$ plot. It is interesting to note the difference between $\langle a(\phi, \psi)\rangle_{\{h k l\}}$ versus $\sin ^{2} \psi$ plots obtained for the studied samples subjected to different surface treatments. In the case of the ground sample, tensile stress caused nonlinearities (Fig. 12) similar to those observed during the tensile test (Fig. 8) and those simulated for a stress of $500 \mathrm{MPa}$ (cf. Fig. 7a). Because of the elastic anisotropy of the crystals, the value of the $\langle a(\phi, \psi)\rangle_{\{111\}}$ parameter is greater, while the value of $\langle a(\phi, \psi)\rangle_{\{200\}}$ is less than the corresponding value on the linear regression line ( $c f$. Fig. $7 a)$. It was found that the shifts in the measured $\langle a(\phi, \psi)\rangle_{\{111\}}$ and $\langle a(\phi, \psi)\rangle_{\{200\}}$ values were always greater than those that could be obtained using

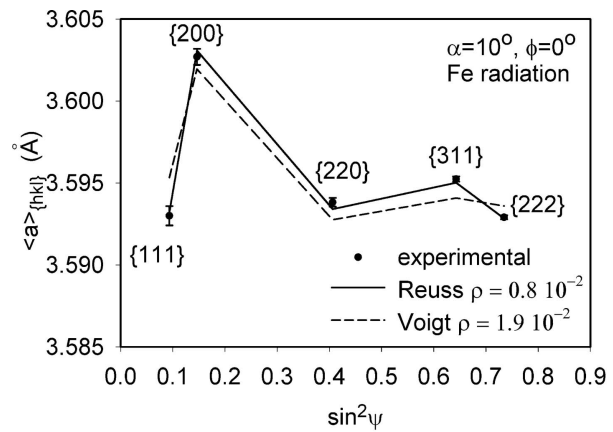

(a)

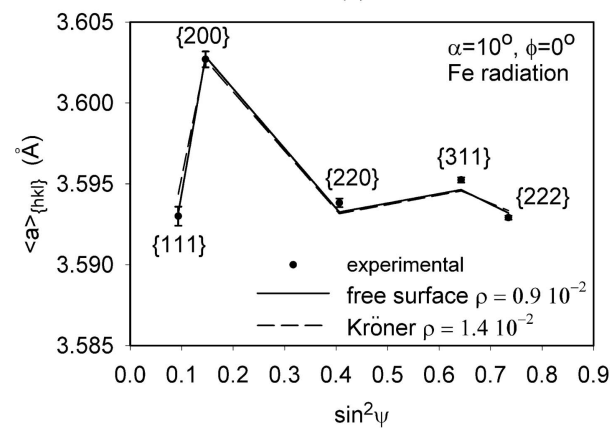

(c)

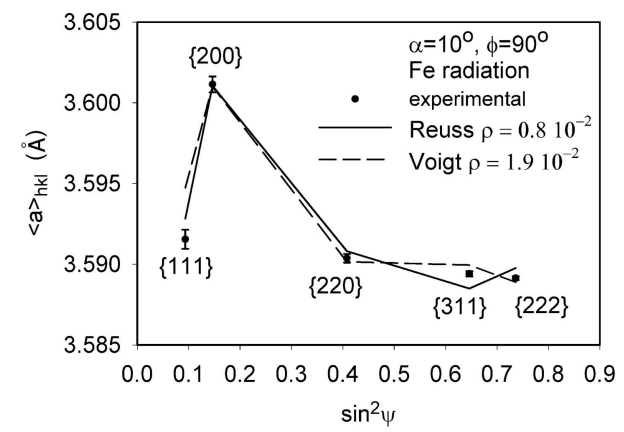

(b)

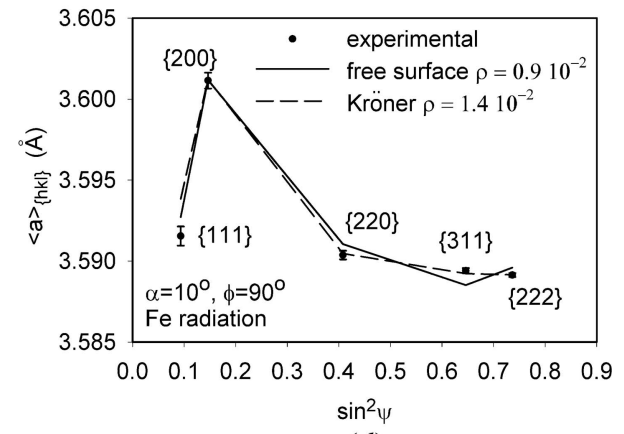

(d)
Figure 15

The $\langle a(\phi, \psi)\rangle_{\{h k l\}}$ lattice parameters fitted to the experimental points for the polished AISI316L sample (Fe $K \alpha$ radiation and $\alpha=10^{\circ}$ ) using equation (10) and different types of XSF models. Plots for $(a),(c) \phi$ $=0^{\circ}$ and for $(b),(d) \phi=90^{\circ}$ are shown. The results of the fitting are listed in Table 5; the uncertainty of the peak position is $\delta(2 \theta)=0.01^{\circ}$. the elastic models (even the Reuss model predicting the largest possible nonlinearities). The opposite shifts in the $\langle a(\phi, \psi)\rangle_{\{111\}}$ and $\langle a(\phi, \psi)\rangle_{\{200\}}$ values were caused by stacking faults (Fig. 7c). Therefore, the theoretical nonlinearities of the $\langle a(\phi, \psi)\rangle_{\{h k l\}}$ versus $\sin ^{2} \psi$ plot decrease and the calculated equivalent lattice parameters approach experimental results when the $\rho$ value is fitted in equation (10) ( $c f$. Fig. 16). In the polished sample, compressive stress decreases the $\langle a(\phi, \psi)\rangle_{\{111\}}$ value and increases the $\langle a(\phi, \psi)\rangle_{\{200\}}$ value $(c f$. Fig. 15) in comparison with the values on the regression line (cf. Fig. 7b). Thus, the effect of elastic anisotropy and the presence of stacking faults shift the values of the $\langle a(\phi, \psi)\rangle_{\{h k l\}}$ parameters significantly in the same direction, increasing the magnitude of nonlinearities (as seen in Fig. 16, the model nonlinearities caused by compressive stress and assuming $\rho=$ 0 are too small in comparison with the experimental ones).

Finally, the influence of crystallographic texture on the determined stress, $a_{0}$ parameter and $\rho$ value was also studied for the ground and polished samples. It was found that this influence is not significant and is at least five times smaller than the experimental uncertainty for all determined values. Since the influence of texture was insignificant, the depthdependent texture gradients should not significantly affect the results obtained for the studied austenitic samples either.

Analysing the results of stress measurement performed for the polished and ground austenitic samples as well as the results of the tensile test $(\$ 4)$, it can be concluded that the Reuss and free-surface grain interaction models lead to the best agreement of the predicted lattice strains with the experimental data. It should be underlined that the application of the free-surface model for the calculation of the XSFs used in the grazing-incidence method can be argued for based on the shallow penetration of the material by $\mathrm{X}$-rays. In this case, grains close to the surface can freely deform in the normal direction, i.e. the assumption of the free-surface model is fulfilled. These models best reflect the elastic anisotropy of the studied samples. It should be also stated that, in previous studies, Krönertype XECs/XSFs were positively verified for quasi-isotropic materials (without texture; e.g. Hauk, 1997) or in some cases for textured samples (Sprauel et al., 1989). However, it was also shown that, in the case of strongly textured samples, the anisotropy of XSFs can be better predicted by the Reuss (Hauk, 1997) or free-surface models (Baczmański et al., 2008). In general, the choice of the correct model for the calculation of the 

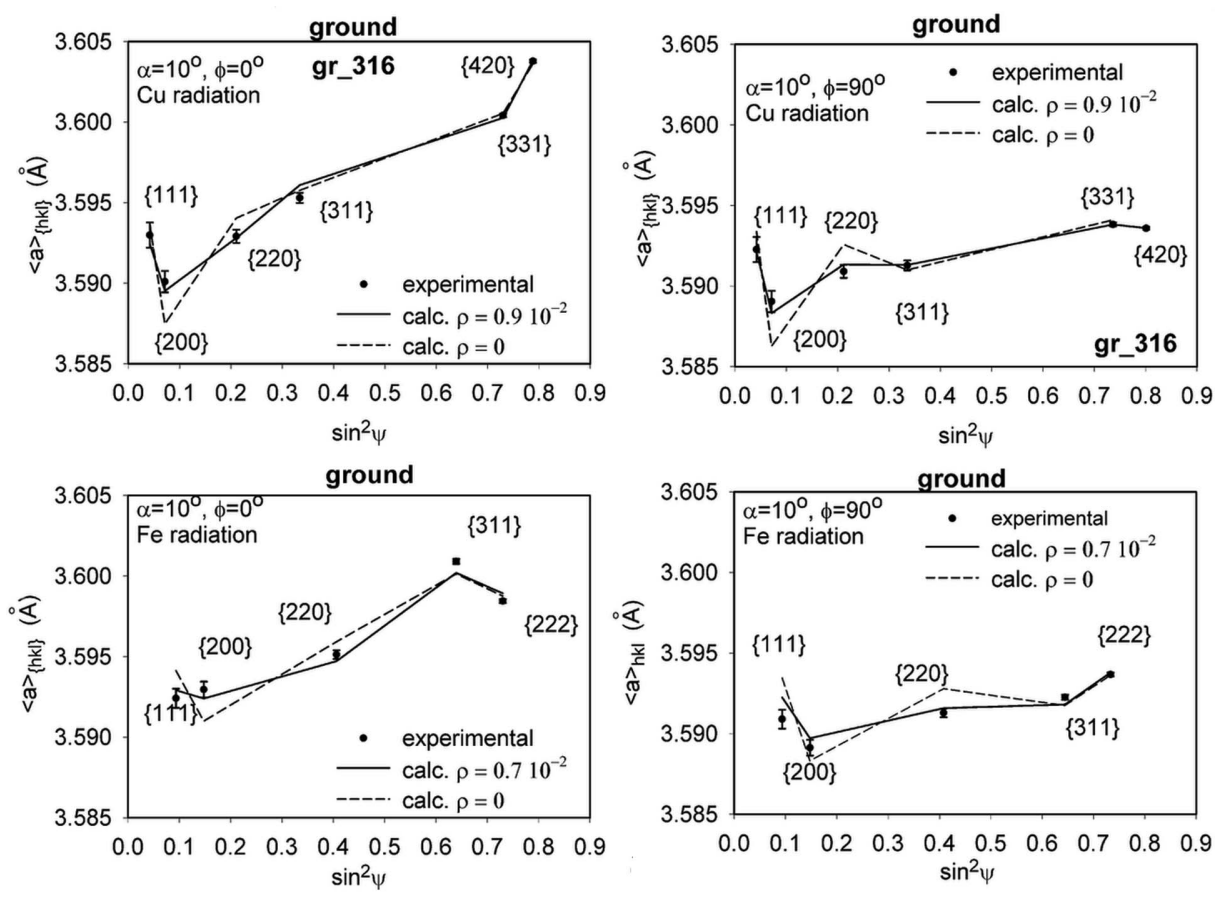

(a)
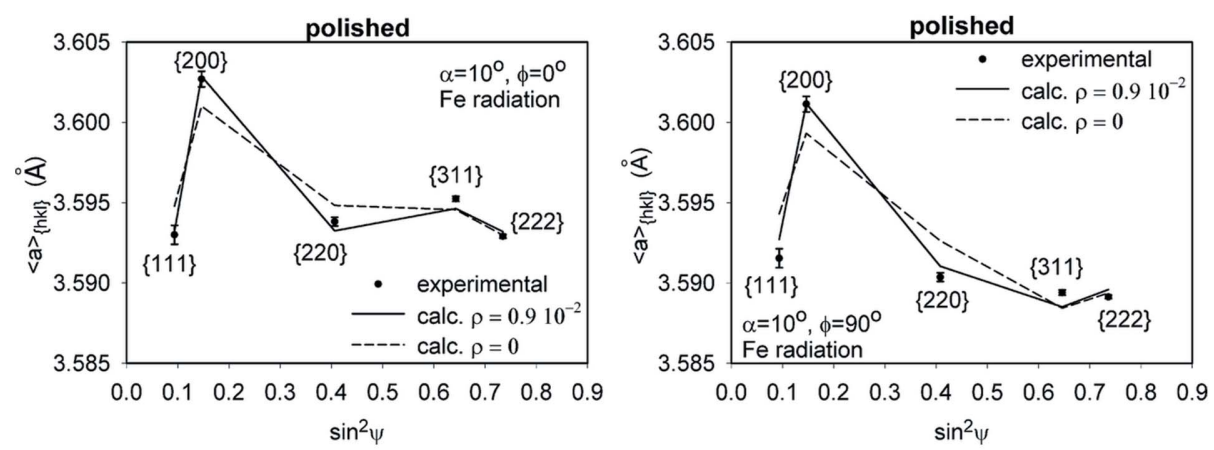

Figure 16

(b)

The $\langle a(\phi, \psi)\rangle_{\{h k l\}}$ lattice parameters fitted to the experimental points using equation (10) (assuming $\rho \neq 0$ - continuous line or $\rho=0$ - dashed line) for the (a) ground and $(b)$ polished AISI316L samples. The free-surface model was used to calculate the XSFs. The uncertainty of the peak position is $\delta(2 \theta)=0.01^{\circ}$.

XSFs is not obvious, especially in the case of a strongly anisotropic sample and/or a small penetration depth of radiation. Therefore, in the case of the MGIXD method (rather small penetration depth), the verification of the model should be performed for an in situ loaded sample, as was done in the present study.

\section{Summary}

In summary, it can be stated that the MGIXD method is an indispensable tool for studying the depth-dependent distribution of stress, the strain-free lattice parameter $\left(a_{0}\right)$ and the probability of stacking faults $(\rho)$ in the surface layers of polycrystalline materials. Nevertheless, the applicability of this method is limited by certain factors, such as the anisotropy of elastic constants. In the case of significant elastic anisotropy (as in austenitic steel), XSF verification should be performed by measuring the relative lattice strains during an in situ tensile test. In this study, the Reuss and free-surface models were positively verified and used for the analysis of residual stress. It was also confirmed that, in the case of isotropic single-crystal constants (as in tungsten), the choice of XSF model does not influence the results of stress analysis.

When the XSFs are known and experimentally verified, new potential information concerning properties of surface layers can be obtained. Here, mechanically polished and ground AISI316L, an austenitic alloy with low stacking fault energy, was studied. It was shown that, in spite of great uncertainty, the variation of the probability of finding stacking faults with the depth below the surface can be determined. Also, a significantly better fit of theoretical data to experimental results can be obtained if the effect of stacking faults is taken into account in the analysis. 
This work was financed by grants from the Polish National Scientific Center (NCN): UMO-2011/03/N/ST8/04058 and DEC-2011/01/D/ST8/07399. Financing by the Polish Ministry of Science and Higher Education (MNiSW) is also acknowledged.

\section{References}

Baczmański, A. (2005). Stress Fields in Polycrystalline Materials Studied Using Diffraction and Self-Consistent Modeling. Kraków: Akademia Górniczo-Hutnicza.

Baczmański, A., Braham, C. \& Seiler, W. (2003). Philos. Mag. 83, 3225-3246.

Baczmański, A., Braham, C., Seiler, W. \& Shiraki, N. (2004). Surf. Coat. Technol. 182, 43-54.

Baczmański, A., Hfaiedh, N., François, M. \& Wierzbanowski, K. (2009). Mater. Sci. Eng. A, 501, 153-165.

Baczmański, A., Lipinski, P., Tidu, A., Wierzbanowski, K. \& Pathiraj, B. (2008). J. Appl. Cryst. 41, 854-867.

Baczmański, A., Wierzbanowski, K., Haije, W. G., Helmholdt, R. B., Ekambaranathan, G. \& Pathiraj, B. (1994). Cryst. Res. Technol. 28, 229-243.

Barral, M., Lebrun, J. L., Sprauel, J. M. \& Maeder, G. (1987). Metall. Trans. A, 18, 1229-1238.

Bollenrath F., Hauk V. \& Muller E. H. (1967). Z. Metallkd. 58, 7682.

Brakman, C. M. (1987). Philos. Mag. A, 55, 39-58.

Brakman, C. M. (1988). PhD thesis, Technische Universiteit Delft, The Netherlands.

Bunge, H. J. (1982). Texture Analysis in Materials Science: Mathematical Methods. London: Butterworths and Co.

Dakhlaoui, R., Baczmański, A., Braham, C., Wroński, S., Wierzbanowski, K. \& Oliver, E. C. (2006). Acta Mater. 54, 5027-5039.

Dölle, H. (1979). J. Appl. Cryst. 12, 489-501.

Dölle, H. \& Cohen, J. B. (1980). Metall. Trans. A, 11, 159-164.

Dölle, H. \& Hauk, V. (1978). Z. Metallkd. 69, 410-417.

Erbacher, T., Wanner, A., Beck, T. \& Vöhringer, O. (2008). J. Appl. Cryst. 41, 377-385.

Eshelby, J. D. (1957). Proc. R. Soc. London Ser. A, 241, 376-396.

Genzel, Ch. (1994). Phys. Status Solidi. (A), 146, 629-637.

Genzel, C., Broda, M., Dantz, D. \& Reimers, W. (1999). J. Appl. Cryst. 32, 779-787.

Hauk, V. (1997). Structural and Residual Stress Analysis by Nondestructive Methods. Amsterdam: Elsevier.

Humphreys, F. J. \& Hatherly, M. (2004). Recrystallisation and Related Annealing Phenomena Oxford: Elsevier Science.

Jegou, S., Christiansen, T. L., Klaus, M., Genzel, Ch. \& Somers, M. A. J. (2013). Thin Solid Films, 530, 71-76.

Kallend, J. S., Kocks, U. F., Rollet, A. D. \& Wenk, H. R. (1990). Report LA-UR-90-2852. Center for Material Sciences, Los Alamos National Laboratory, New Mexico, USA.

Klaus, M. \& Genzel, Ch. (2013). J. Appl. Cryst. 46, 1266-1276.

Kröner, E. (1961). Acta Metall. 9, 155-161.

Kumar, A., Welzel, U. \& Mittemeijer, E. J. (2006). J. Appl. Cryst. 39, 633-646.

Leeuwen, M. van, Kamminga, J.-D. \& Mittemeijer, E. J. (1999). J. Appl. Phys. 86, 1904-1914.

Lipinski, P. \& Berveiller, M. (1989). Int. J. Plasticity, 5, 149-172.
Malkin, S. \& Guo, C. (2007). CIRP Ann. Manuf. Technol. 56, 760782.

Marciszko, M., Baczmański, A., Wierzbanowski, K., Wróbel, M., Braham, C., Chopart, J.-P., Lodini, A., Bonarski, J., Tarkowski, L. \& Zazi, N. (2013). Appl. Surf. Sci. 266, 256-267.

Marciszko, M., Baczmański, A., Wróbel, M., Seiler, W., Braham, C., Donges, J., Śniechowski, M. \& Wierzbanowski, K. (2013). Thin Solid Films, 530, 81-84.

Marques, M. J., Pina, J. C. P. \& Dias, A. M. (2006). Mater. Sci. Forum, 514-516, 1618-1622.

Matthies S. \& Humbert M. (1995). J. Appl. Cryst. 28, 254-266.

Noyan, I. C. \& Cohen, J. B. (1987). Residual Stress. Measurement by Diffraction and Interpretation. New York: Springer-Verlag.

Ortner, B. (2005). Z. Metallkd. 96, 1049-1055.

Ortner, B. (2006). J. Appl. Cryst. 39, 401-409.

Paterson, M. S. (1952). J. Appl. Phys. 23, 805-811.

Predecki, P., Ballard, B. \& Zhu, X. (1993). Adv. X-ray Anal. 36, $237-$ 245.

Press, W. H., Flannery, B. P., Teukolsky, S. A. \& Vetterling, W. T. (1989). Numerical Recipes. The Art of Scientific Computing. Cambridge University Press.

Reimers, W., Pyzalla, A. R., Schreyer, A. \& Clemens, H. (2008). Neutrons and Synchrotron Radiation in Engineering Materials Science. Weinheim: Wiley-VCH.

Reuss, A. (1929). Z. Angew. Math. Mech. 9, 49-58.

Ruppersberg, H., Detemple, I. \& Krier, J. (1989). Phys. Status Solidi (A), 116, 681-687.

Schuman, C., Humbert, M. \& Esling, C. (1994). Z. Metallkd. 85, 559_ 563.

Simmons, G. \& Wang, H. (1971). Single Crystal Elastic Constants and Calculated Aggregate Properties: a Handbook, 2nd ed. Cambridge, London: MIT Press.

Skrzypek, S. J. \& Baczmański, A. (2001). Adv. X-ray Anal. 44, 134 145.

Skrzypek, S. J., Baczmański, A., Ratuszek, W. \& Kusior, E. (2001). J. Appl. Cryst. 34, 427-435.

Sprauel, J. M., Francois, M. \& Barral, M. (1989). Proceedings of the 2nd International Conference on Residual Stresses ICRS2, edited by G. Beck, S. Denis \& A. Simon. London, New York: Elsevier Applied Science.

Van Acker, K., De Buyser, L., Celis, J. P. \& Van Houtte, P. (1994). J. Appl. Cryst. 27, 56-66.

Velterop, L., Delhez, R., de Keijser, Th. H., Mittemeijer, E. J. \& Reefman, D. (2000). J. Appl. Cryst. 33, 296-306.

Voigt, W. (1928). Lehrbuch der Kristallphysik. Leipzig: BG Teubner.

Wagner, C. N. J. (1957). Acta Metall. 5, 427-434.

Wagner, C. N. J. (1966). Metall. Soc. Conf. 36, 219.

Warren, B. E. (1990). X-ray Diffraction. New York: Dover Publications.

Warren, B. E. \& Warekois, E. P. (1955). Acta Metall. 3, 473-479.

Wawszczak, R., Baczmański, A., Braham, C., Seiler, W., Wróbel, M., Wierzbanowski, K. \& Lodini, A. (2011). Philos. Mag. 91, 2263 2290.

Welzel, U., Leoni, M. \& Mittemeijer, E. J. (2003). Philos. Mag. 83, 603-630.

Welzel, U., Ligot, J., Lamparter, P., Vermeulen, A. C. \& Mittemeijer, E. J. (2005). J. Appl. Cryst. 38, 1-29.

Witt, F. \& Vook, R. W. (1968). J. Appl. Phys. 39, 2773-2776.

Wroński, S., Baczmański, A., Dakhlaoui, R., Braham, C., Wierzbanowski, K. \& Oliver, E. C. (2007). Acta Mater. 55, 62196233 . 